\title{
Multiple black hole system in 4C31.61 (2201+315)
}

\author{
J. Roland ${ }^{1}$, C. Gattano ${ }^{2}$, S. B. Lambert ${ }^{3}$, and F. Taris ${ }^{3}$ \\ 1 Institut d'Astrophysique, UPMC Univ. Paris 06, CNRS, UMR 7095, 98 bis Bd Arago, 75014 Paris, France \\ e-mail: roland@iap.fr \\ 2 Laboratoire d'Astrophysique de Bordeaux, Univ. Bordeaux, CNRS, B18N, Allée Geoffroy Saint-Hilaire, 33615 Pessac, France \\ 3 SYRTE, Observatoire de Paris, Université PSL, CNRS, Sorbonne Université, LNE, France
}

Received 29 November 2018 / Accepted 11 December 2019

\begin{abstract}
Modeling trajectories of radio components ejected by the nucleus of 4C31.61 $(2201+315)$ and observed by very long baseline interferometry (VLBI) in the frame of the MOJAVE survey suggests that they are ejected from three different origins that possibly host three different supermassive black holes. These origins correspond to three stationary components, one of which one is the VLBI core. Most of the mass of the nucleus is associated with a supermassive binary black hole system whose separation is $\approx 0.3$ milliarc second, that is, a distance of $\approx 1.3$ parsec and the mass ratio is $\approx 2$. In contrast, the mass ratio with respect to the third black hole is $\approx 1 / 100$. The three origins lie within 0.6 milliarc second, or a distance of $\approx 2.6$ parsec. Based in this structure of the nucleus, we explain the variations observed in the astrometric coordinate time series obtained from VLBI geodetic surveys. This study shows that it is possible to exploit large MOJAVE-like VLBI databases to propose more insights into the structure of the extragalactic radio sources that are targeted by VLBI in geodetic and astrometry programs.
\end{abstract}

Key words. astrometry - galaxies: jets - galaxies: individual: $2201+315$

\section{Introduction}

Very long baseline interferometry (VLBI) observations of nuclei of extra galactic radio sources show that the ejection of VLBI components does not follow a straight line, but wiggles. These observations suggest a precession of the accretion disk that can be explained if the nuclei contain either a spinning single black hole $(\mathrm{BH})$ or a binary black hole $(\mathrm{BBH})$ system. We developed a minimization method by first modeling the ejection of a VLBI component by a spinning single $\mathrm{BH}$, which produces the precession of the accretion disk. Then we modeled the ejection of a VLBI component by a BBH system, taking the precession of the accretion disk and the motion of the BHs into account.

The method has been applied to 0420-014 (Britzen et al. 2001), 3C 345 (Lobanov \& Roland 2005), 1803+784 (Roland et al. 2008), 1823+568 and 3C 279 (Roland et al. 2013), and $1928+738$ (Roland et al. 2015). Modeling VLBI ejections produces evidence that nuclei of extragalactic radio sources contain BBH systems. These systems can form when galaxies merge (Begelman et al. 1980), and Britzen et al. (2001) suggested that if nuclei of extragalactic radio sources contain $\mathrm{BBH}$ systems, the association of extragalactic radio sources with elliptical galaxies can be explained. It also explains why quasars (quasi-stellar radio sources) represent about $5 \%$ of the quasi-stellar objects (QSO). For reviews for the formation of BBH systems in galaxy mergers, see Colpi \& Dotti (2011), Colpi (2014), and Volonteri et al. (2016).

When a nucleus contains a BBH system, the two BHs can eject VLBI components, and both can be detected using VLBI observations. In this case, we observe the core and one stationary component, and we observe two families of trajectories. This is, for instance, the case for 3C 279 (Roland et al. 2013) and $1928+738$ (Roland et al. 2015). However, it is not always the case, and in some cases, only one BH can be detected using VLBI observations, the other being "inactive".

In this article, we use for the Hubble constant $H_{\mathrm{o}} \approx$ $73 \mathrm{~km} \mathrm{~s}^{-1} \mathrm{Mpc}^{-1}$ and $D_{\mathrm{L}} \approx 1460 \mathrm{Mpc}$ for the luminosity distance of $2201+315$ and model the ejection of the VLBI components of $4 \mathrm{C} 31.61 \equiv 2201+315$ using data from the Monitoring Of Jets in Active galactic nuclei with VLBA Experiments (MOJAVE), that is, the $15 \mathrm{GHz}$ observations and the definitions of the different components of Table 3 of Lister et al. (2019). The analysis of MOJAVE data with our minimization method suggests that the nucleus of $2201+315$ contains three BHs associated with the stationary components $\mathrm{C} 0$ (the core), C3, and C9 (see Fig. 1). Most of the mass of the nucleus is associated with the BBH system C0-C9, whose separation is $R_{\mathrm{C} 0-\mathrm{C} 9}=0.296_{-0.020}^{+0.066}$ mas, corresponding to a projected linear distance $=1.3_{-0.09}^{+0.26} \mathrm{pc}$, and the mass ratio is $M_{\mathrm{C} 9} / M_{\mathrm{CO}}=2 \pm 0.5$. The mass of the third $\mathrm{BH}$ is $M_{\mathrm{C} 3} /\left(M_{\mathrm{C} 0}+M_{\mathrm{C} 9}\right) \approx 1 / 100$. The distance between $\mathrm{C} 0$ and $\mathrm{C} 3$ is $R_{\mathrm{C} 0-\mathrm{C} 3}=0.557_{-0.124}^{+0.124}$ mas, which translates into $\approx 2.4 \mathrm{pc}$. Calling $\mathrm{G}$, the center of gravity of the system $\mathrm{C} 0-\mathrm{C} 9$, the distance between component $\mathrm{C} 3$ and the center of gravity $\mathrm{G}$ is $R_{\mathrm{G}-\mathrm{C} 3}=0.369_{-0.124}^{+0.124}$ mas, that is, $\approx 1.6 \mathrm{pc}$ (see Table 1 ).

We show that component $\mathrm{C} 6$ is ejected from the core $\mathrm{C} 0$, component $\mathrm{C} 7$ is ejected from the stationary component $\mathrm{C} 3$, and component $\mathrm{C} 13$ is ejected from the stationary component $\mathrm{C}$. We deduce the characteristics of the $\mathrm{BBH}$ system constituted by the core $\mathrm{C} 0$ and the stationary component $\mathrm{C} 9$. We also deduce the characteristics of the third $\mathrm{BH}$ that is associated with the stationary component $\mathrm{C} 3$. Finally, knowing the structure of the nucleus of $2201+315$, we discuss the influence of the variations in the ratio of the flux densities of the three BHs and of the ejection of a new VLBI component on the coordinate time series obtained by the analysis of geodetic VLBI observations (Gattano et al. 2018). 
Table 1. Distances between the different BH.

\begin{tabular}{lc}
\hline \hline System & Separation \\
\hline $\mathrm{C} 0-\mathrm{C} 9$ & $R_{\mathrm{C} 0-\mathrm{C} 9}=0.296_{-0.020}^{+0.066}$ mas $\approx 1.3 \mathrm{pc}$ \\
$\mathrm{C} 0-\mathrm{C} 3$ & $R_{\mathrm{C} 0-\mathrm{C} 3}=0.577_{-0.124}^{+0.124}$ mas $\approx 2.4 \mathrm{pc}$ \\
\hline
\end{tabular}

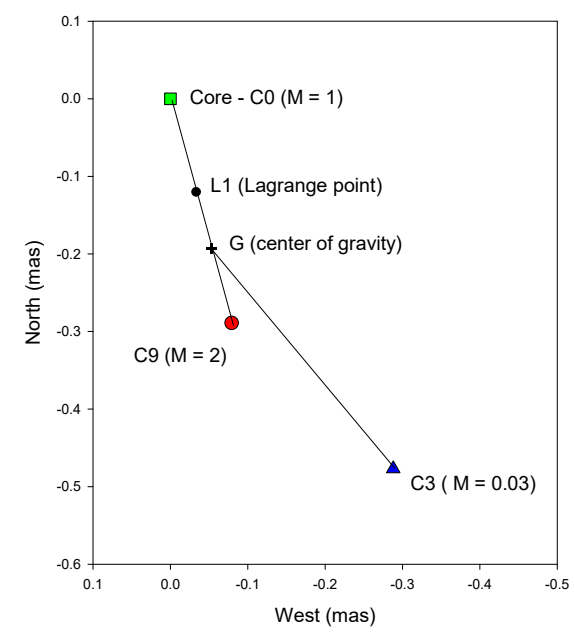

Fig. 1. Structure of the nucleus of $2201+315$. The analysis of MOJAVE data with our minimization method suggests that the nucleus of $2201+315$ contains three BHs. G is the center of gravity of the BBH system $\mathrm{C} 0-\mathrm{C} 9$ and $\mathrm{L} 1$ is the first Lagrange point of the $\mathrm{BBH}$ system $\mathrm{C} 0-\mathrm{C}$; this is the point where the gravitational forces of the $\mathrm{BHs} \mathrm{C} 0$ and $\mathrm{C} 9$ are equal. In order to obtain a stable solution, the radii of the accretion disks around $\mathrm{C} 0$ and $\mathrm{C} 9$ have to be smaller than the distances L1-C0 and L1-C9, respectively.

\section{Methods and data}

\subsection{Properties of the $B B H$ system solution}

To model the ejection of a VLBI component, we assumed that the VLBI component corresponds to the relativistic ejection of an electron-positron $\left(\mathrm{e}^{-} \mathrm{e}^{+}\right)$plasma referred to as the beam. It is surrounded by a subrelativistic electron-proton $\left(e^{-} p\right)$ plasma called the jet. The relativistic beam is responsible for the formation of VLBI components, their synchrotron radio emission, and inverse Compton emission in the UV, X-rays, and $\gamma$-rays. The subrelativistic electron-proton jet that carries most of the mass and kinetic energy ejected by the $\mathrm{BH}$ is responsible for the formation of kiloparsec jets, hot spots, and extended lobes. The magnetic field in the beam and the mixing layer between the beam and the jet is parallel to the flow, and the magnetic field in the jet rapidly becomes toroidal. This model is called the twofluid model (Fig. 2; see also Roland et al. 2013, and references therein). The beam can propagate along the magnetic lines and is stable if the bulk Lorentz factor, $\gamma_{\mathrm{c}}$ of the ejected VLBI component is $\gamma_{\mathrm{c}}<30$ and if the magnetic field in the beam and in the mixing layer is grater than a critical value $B_{\mathrm{c}}$. The beam and the jet are perturbed by the precession of the accretion disk and the motion of the BHs.

When in addition to the radio, optical observations are available that peak in the light curve, this optical emission can be modeled as the synchrotron emission of a point source ejected in the perturbed beam, see Britzen et al. (2001) and Lobanov \& Roland (2005). This short burst of very energetic, relativistic $\mathrm{e}^{ \pm}$plasma is followed immediately by a very long burst of less energetic relativistic $\mathrm{e}^{ \pm}$plasma. This long burst is modeled as

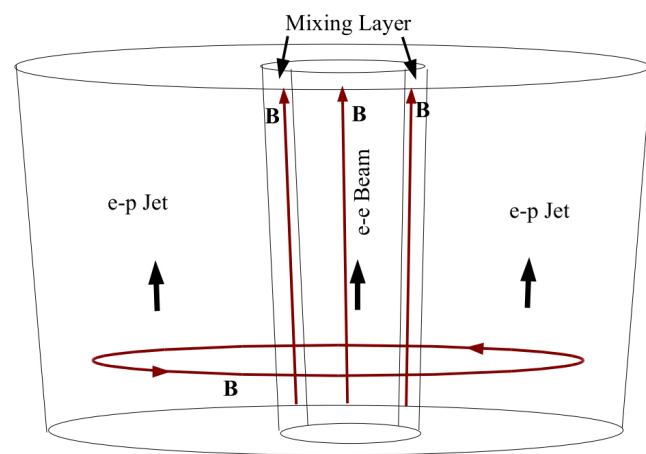

Fig. 2. Two-fluid model. The outflow consists of an $e^{-}-e^{+}$plasma, the beam, which moves at a highly relativistic speed and is surrounded by an $e^{-}-p$ plasma, and of the jet, which moves at a mildly relativistic speed.

an extended structure along the beam and is responsible for the VLBI radio emission.

In the case of ejection by a spinning single $\mathrm{BH}$, we found in general that the surface $\chi^{2}$ of the solution that corresponds to the inclination angle is convex, indicating that there is no stable solution. However, we found that it becomes concave when the ejection is caused by a BBH system. For details on the model geometry, the perturbation of the VLBI ejected component, and the coordinates of the ejected VLBI component, see Appendix A.

When the two BHs eject VLBI components, we observe two families of trajectories. We find by fitting the ejection of VLBI components of the two families that if one family is characterized by the mass ratio $M_{1} / M_{2}=a$, where $M_{1}$ and $M_{2}$ are the masses of the two BHs, the second family is characterized by the inverse mass ratio $1 / a$, showing the consistency of the $\mathrm{BBH}$ model. The solution of the fit is not unique and shows a degeneracy; the degeneracy parameter being $V_{\mathrm{a}}$, the propagation speed of the perturbation of the jet and the beam. This means that $\chi^{2}\left(V_{\mathrm{a}}\right)=$ constant, that is, $\chi^{2}\left(V_{\mathrm{a}}\right)$ does not depend on the value of $V_{\mathrm{a}}$ when $V_{\mathrm{a}}$ varies. It also means that there is a possible range of values for the $\mathrm{BBH}$ system period $T_{\mathrm{b}}$ and the $\mathrm{BBH}$ system mass $M_{1}+M_{2}$.

\subsection{Properties of the radio source $2201+315$}

The source has been observed using the Very Long Baseline Array (VLBA) in the frame of the MOJAVE survey ${ }^{1}$ at $15 \mathrm{GHz}$ (Lister et al. 2019) and at 43 and $86 \mathrm{GHz}$ (Cheng et al. 2018). The positions of the components $\mathrm{J} 1, \mathrm{~J} 2$, and $\mathrm{J} 3$ detected at 43 and $86 \mathrm{GHz}$ are shown in Fig. 8. The position of $2201+315$ provided by the MOJAVE survey is shown in Figs. 3 and 4 with the stacked $15 \mathrm{GHz}$ map and a plot of separation versus time, with the component names as provided by the MOJAVE survey. These observations reveal the stationary radio components $\mathrm{C} 3$ and $\mathrm{C} 9$, whose flux densities are variable with time. We show that these components can be linked with the BHs that eject VLBI components. Source $2201+315$ has been observed during more than 20 years by MOJAVE, and an important property of these components is that they are detected during different periods of time.

Radio source $2201+315^{2}$ is associated with a quasar whose active galactic nucleus (AGN) class is low-spectral peak

\footnotetext{
1 See details at http://WwW.physics.purdue.edu/astro/ MOJAVE

2 Also known as 4C+31.63, B2 2201+31A, J220314.9+314538, or
} CGRaBS J2203+3145. 


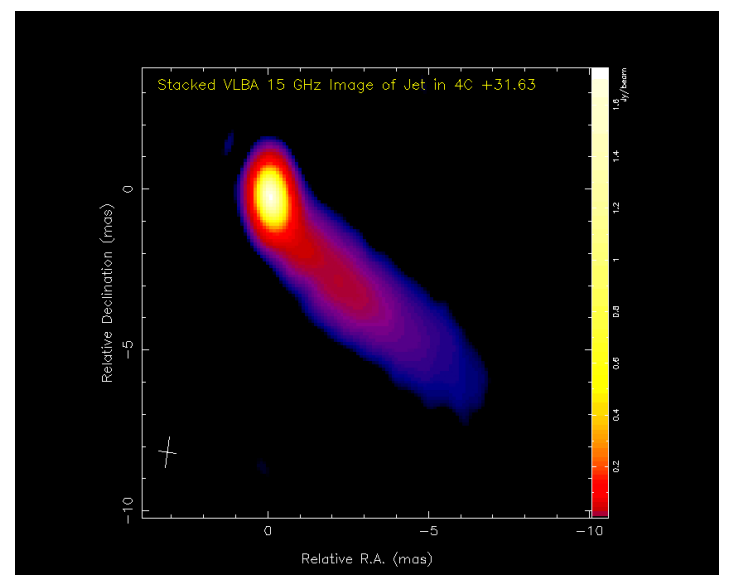

Fig. 3. $15 \mathrm{GHz}$ map of $2201+315$ provided in the MOJAVE survey.

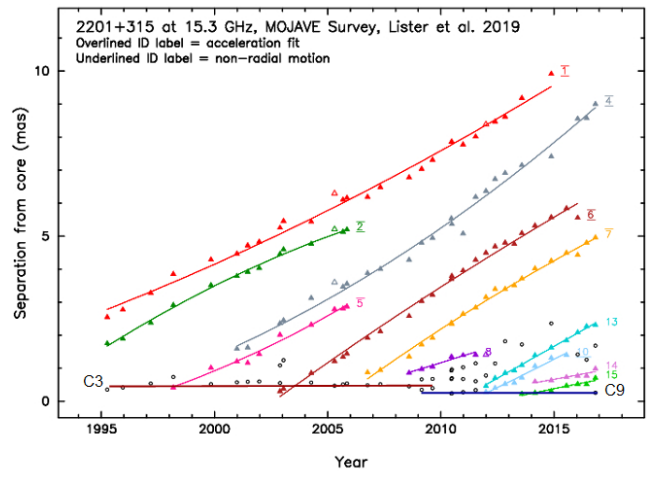

Fig. 4. Stationary components $\mathrm{C} 3$ and $\mathrm{C} 9$ added to the plot of separation vs. time provided in the MOJAVE survey (Lister et al. 2019).

$<10^{14} \mathrm{~Hz}$ (LSP), and it is a quasar whose fractional linear optical polarization is consistently below $3 \%$ (LQP). Its redshift is $z \approx 0.2947$ according to Ho \& Kim (2009). Hutchings \& Morris (1995) reported that Canada France Hawaii Telescope (CFHT) $3.6 \mathrm{~m}$ optical telescope images of $2201+315$ indicated an overall elliptical shape, extended normal to the radio axis, but with a radial color gradient (redder with increasing distance), and some irregular knots within the host galaxy. The total magnitudes (R15.6 and V16.6) are close to those quoted in Hewitt \& Burbidge (1993) and Hutchings \& Neff (1992), and the small differences are probably due to differing extensions of the host galaxy that were measured. Healey et al. (2008) reported an Rmag of $M_{\mathrm{R}} \approx 14.33$. The Gaia DR2 catalog (Brown et al. 2018) gives a Gmag of 15.3969, a GBPmag of 15.4428, and a GRPmag of 14.9321 (Brown et al. 2018). Gmag (wavelength range 330$1050 \mathrm{~nm}$ ), GBPmag (blue photometer, wavelength range 330 $680 \mathrm{~nm}$ ) and GRPmag (red photometer, wavelength range 640 $1050 \mathrm{~nm}$ ) are Gaia magnitudes defined in Carrasco et al. (2016). The source has also been detected by the Fermi/LAT - $\gamma$-ray space telescope (Acero et al. 2015). It is a strong X-ray AGN observed by Swift (Maselli et al. 2010).

\subsection{Stationary component $\mathrm{C3}$}

The stationary component $\mathrm{C} 3$ is detected at $15 \mathrm{GHz}$ between 1995 and 2011 (Lister et al. 2019). The mean position of C3 is $X_{\mathrm{C} 3}=-0.288 \pm 0.055$ mas and $Y_{\mathrm{C} 3}=-0.477 \pm 0.111 \mathrm{mas}$ (see Fig. 1), and the distance between cores $\mathrm{C} 0$ and $\mathrm{C} 3$ is $R_{\mathrm{C} 3-\mathrm{C} 0}=0.557 \pm 0.124$ mas (see Fig. 4 ). The flux density of

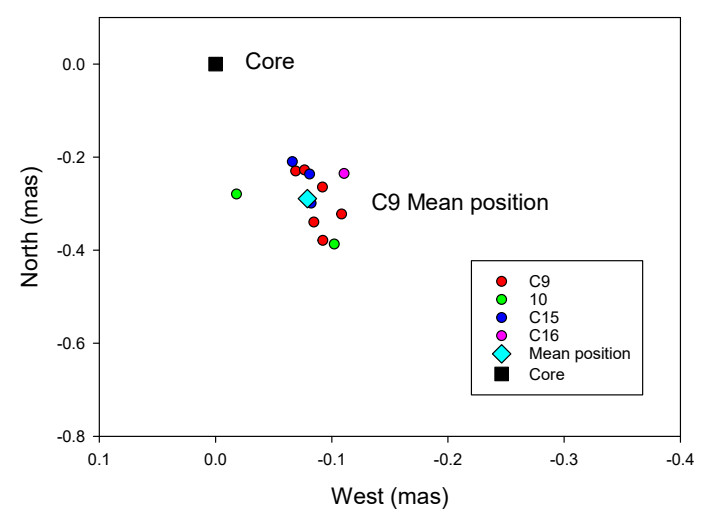

Fig. 5. Stationary component C9, identified and observed between 2009 and 2011 (Lister et al. 2019). However, identification of the first points of other components indicates that the two first points of component $\mathrm{C} 10$, the three first points of component $\mathrm{C} 15$, and component $\mathrm{C} 16$, which are located at the same position as component $\mathrm{C} 9$, are probably associated with component $\mathrm{C}$.

$\mathrm{C} 3$ at $15 \mathrm{GHz}$ is variable, but weaker than or equal to the flux density of the core (see Fig. 10). We show that component C3 is responsible for the ejection of component $\mathrm{C} 7$.

\subsection{Stationary component C9}

The stationary component C9 has been identified and observed between 2009 and 2011 (Lister et al. 2019). However, the identification of the first points of other components indicates that the first two points of component $\mathrm{C} 10$, the first three points of component $\mathrm{C} 15$, and component $\mathrm{C} 16$, which are located at the same position as component $\mathrm{C} 9$, are probably associated with component C9 (see Figs. 4 and 5). This means that component C9 is detected between 2009 and 2017.

The mean position of $\mathrm{C} 9$ is $X_{\mathrm{C} 9}=-0.082 \pm 0.025$ mas and $Y_{\mathrm{C} 9}=-0.284 \pm 0.061$ mas (see Figs. 1 and 5) and the distance between cores $\mathrm{C} 0$ and $\mathrm{C} 9$ should be $R_{\mathrm{C} 0-\mathrm{C} 9}=0.296 \pm 0.066$ mas. However, the error on the distance between cores $\mathrm{C} 0$ and $\mathrm{C} 9$ is not symmetrical because of the condition $R_{\text {disk,C0 }}<D_{\mathrm{L} 1-\mathrm{C} 0}$ (see Eq. (3) and Appendix B.2.1), and we adopt $R_{\mathrm{C} 0-\mathrm{C} 9}=$ $0.296_{-0.020}^{+0.066}$ mas (see Fig. 4) for the distance between the core and $\mathrm{C} 9$. A better determination of the distance between $\mathrm{C} 0$ and C9 and its error bars could be achieved during the fit of component C6 (see Appendix B.2.1), but this is beyond the scope of this article. Its flux density at $15 \mathrm{GHz}$ is variable but often higher than the flux density of the core (see Fig. 12). It is detected at $15 \mathrm{GHz}$ between 2009 and 2017.

We show that component $\mathrm{C} 13$ is ejected from the stationary component $\mathrm{C} 9$. Components $\mathrm{C} 10, \mathrm{C} 15$, and $\mathrm{C} 17$ are probably also ejected by the $\mathrm{BH}$ associated with component $\mathrm{C} 9$.

\subsection{Components used to fit the model}

We fit the precession model and the BBH model parameters using components $\mathrm{C} 6, \mathrm{C} 7$, and $\mathrm{C} 13$, that is, components that on the one hand, have enough observed points to have a welldefined trajectory, and on the other hand, have observed points in the first two or three mas to precisely define the origin of the component. We therefore did not fit components $\mathrm{C} 1, \mathrm{C} 2$, and $\mathrm{C} 4$, which have no observations in the first 3 mas $(\mathrm{C} 1)$ and in the first 2 mas ( $\mathrm{C} 2$ and $\mathrm{C} 4)$. Component $\mathrm{C} 5$, which has a poorly defined trajectory, has not been fit either. 
The observations at $15 \mathrm{GHz}$ that we used for this study correspond to 37 epochs and are reported in Table 3 of Lister et al. (2019). We used the model fit data of Lister et al. (2019) to fit the coordinates $X(t)$ and $Y(t)$ of components $\mathrm{C} 6, \mathrm{C} 7$, and $\mathrm{C} 13$ using the precession and the $\mathrm{BBH}$ model. The results of the fits of components C6, C13, and C7 are given in Sects. 3.4, 3.5, and 3.6, respectively. The positions of the stationary components $\mathrm{C} 0$, $\mathrm{C} 9$, and $\mathrm{C} 3$, the positions of the moving components $\mathrm{C} 6, \mathrm{C}$, and $\mathrm{C} 13$, and the trajectories obtained from the fit of the BBH system are shown in Fig. 7.

\section{Structure of the nucleus of $2201+315$}

\subsection{Stability condition}

We describe an important constraint that can be used to fit an ejected VLBI component below. To obtain a stable solution in a BBH system, for instance, the BBH system C0-C9 (see Fig. 1), the radii of the accretion disks around $\mathrm{C} 0$ and $\mathrm{C} 9$, that is, $R_{\text {disk,C0 }}$ and $R_{\text {disk,C } 9}$, must be smaller than the distances $D_{\mathrm{L} 1-\mathrm{C} 0}$ and $D_{\mathrm{L} 1-\mathrm{C} 9}$, respectively. Here L1 is the first Lagrange point of the $\mathrm{BBH}$ system (the point where the gravitation forces of $\mathrm{C} 0$ and $\mathrm{C} 9$ are equal).

Calling $T_{\mathrm{b}}$ the orbital period and $T_{\mathrm{p}}$ the precession period of the accretion disk, we can calculate the mass of the ejecting $\mathrm{BH}$ $M_{\mathrm{C} 0}, T_{\mathrm{b}}$, and $T_{\mathrm{p}}$ for each value of $V_{\mathrm{a}}$ the propagation speed of the perturbation along the jet and the beam based on the knowledge of the mass ratio $M_{\mathrm{C} 0} / M_{\mathrm{C} 9}$ and the ratio $T_{\mathrm{p}} / T_{\mathrm{b}}$.

The rotation period of the accretion disk around $\mathrm{C} 0, T_{\text {disk,C} 0}$, is given by (Britzen et al. 2001)

$T_{\text {disk,C } 0} \approx \frac{4}{3} \frac{M_{\mathrm{C} 0}+M_{\mathrm{C} 9}}{M_{\mathrm{C} 9}} T_{\mathrm{b}} \frac{T_{\mathrm{b}}}{T_{\mathrm{p}}}$.

When we assume that the mass of the accretion disk is $M_{\text {disk,C } 0} \ll M_{\mathrm{C} 0}$, the radius of the accretion disk $R_{\text {disk,C } 0}$ is

$R_{\mathrm{disk}, \mathrm{C} 0} \approx\left(\frac{T_{\mathrm{disk}, \mathrm{C} 0}^{2}}{4 \pi^{2}} G M_{\mathrm{C} 0}\right)^{1 / 3}$,

and we must have the stability condition

$R_{\text {disk,C0 }}<D_{\mathrm{L} 1-\mathrm{C} 0}=R_{\mathrm{CO}-\mathrm{C} 9} /\left(1+\sqrt{M_{\mathrm{C} 9} / M_{\mathrm{C} 0}}\right)$,

where $D_{\mathrm{L} 1-\mathrm{C} 0}$ is the distance between $\mathrm{C} 0$ and the first Lagrange point $\mathrm{L} 1$, and $R_{\mathrm{C} 0-\mathrm{C} 9}$ is the separation of the $\mathrm{BBH}$ system C0-C9.

The radius of the accretion disk does not depend on $V_{\mathrm{a}}$, which is the propagation speed of the perturbation along the beam and the jet.

\subsection{Separation of the BBH systems}

At the VLBI core, the VLBI jet becomes transparent to a given synchrotron frequency. The position of the VLBI core does not correspond to the position of the supermassive $\mathrm{BH}(\mathrm{SMBH})$, and the distance between the VLBI core and the SMBH depends on the observational frequency. At higher frequency, the VLBI core is closer to the SMBH; see, for instance, Marscher et al. (2008). Although the exact dependence of the distance of the VLBI core to the SMBH with the frequency of observation is not known, the distance between the two VLBI cores that are detected at the same frequency provides a good estimate of the distance between the two SMBH when the ejection directions of
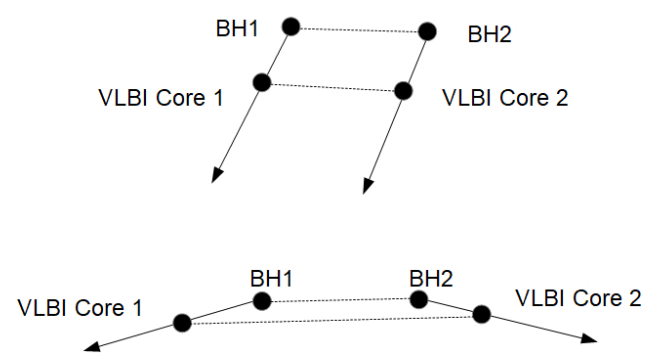

Fig. 6. Top: the distance between the two VLBI cores that are detected at the same frequency provides a good estimate of the distance between the two SMBH, BH1 and $\mathrm{BH} 2$, if ejection directions of the VLBI jets are equal to within few degrees. Bottom: when the jets are ejected in opposite directions or in very different directions, then the distance between the two VLBI cores is very different than the distance between the two SMBH.

the VLBI jets are equal to within few degrees (see Fig. 6). This effect will produce an additional error on the distance determination of the two $\mathrm{BH}$. If the distance between the core and the $\mathrm{BH}$ is about $25 \mu \mathrm{as}$ and the angle between the two jets is $3^{\circ}$, the additional error is $\approx 2 \times 25 \times \sin \left(3^{\circ}\right)=2.6 \mu$ as. When the jets are ejected in opposite directions or in very different directions, the distance between the two VLBI cores is very different than the distance between the two SMBH (see Fig. 6).

\subsection{Parameter ranges we explored for the fit}

In this section, we provide the parameter ranges we explored to fit the VLBI components $\mathrm{C} 6, \mathrm{C} 7$, and $\mathrm{C} 13$. In order to cover a wide range of possible inclination angles, we explored the range $2^{\circ} \leq i_{\mathrm{o}} \leq 40^{\circ}$. For the mass ratio of the $\mathrm{BBH}$ system, we explored the range $10^{-7} \leq M_{1} / M_{2} \leq 10$, where $M_{1}$ is the mass of the $\mathrm{BH}$ ejecting the VLBI component. For the ratio $T_{\mathrm{p}} / T_{\mathrm{b}}$, we explored the range $1 \leq T_{\mathrm{p}} / T_{\mathrm{b}} \leq 10^{4}$. For the propagation speed of the perturbation, we explored the range $0.001 c \leq V_{\mathrm{a}} \leq 0.45 \mathrm{c}$ (we limit ourselves to nonrelativistic hydrodynamics in this model).

\subsection{Fit of component C6}

\subsubsection{Introduction}

We studied the three different models given in Table 2. We calculated the curve $\chi^{2}\left(i_{0}\right)$, which corresponds to the $\chi^{2}$ of the model of the VLBI component ejection for a given inclination angle $i_{\mathrm{o}}$. The details of the calculations corresponding to the three models are given in Appendix B. In this section, we present the results of fitting the ejection of component $\mathrm{C} 6$ from the origin $\mathrm{C} 0$ by the $\mathrm{BBH}$ system $\mathrm{C} 0-\mathrm{C} 9$.

We found that component $\mathrm{C} 6$ cannot be ejected by a single spinning $\mathrm{BH}$ but must be ejected by a BBH system.

\subsubsection{BBH system $\mathrm{CO}-\mathrm{C} 9$ : Origin $\mathrm{CO}$}

The BBH system is constituted by core $\mathrm{C} 0$ and the stationary component C9. Its separation is $R_{\mathrm{C} 0-\mathrm{C} 9}=0.3$ mas. Component C6 is ejected by the VLBI core, that is, component C0 (see Fig. 7).

We found that the mass ratio $M_{\mathrm{C} 0} / M_{\mathrm{C} 9}$ is $0.1 \leq M_{\mathrm{C} 0} /$ $M_{\mathrm{C} 9}<1$ (see Fig. B.3). Using the stability condition, $R_{\text {disk,C } 0}<$ $D(\mathrm{~L} 1-\mathrm{C} 0)$, we found that solutions with the mass ratio $M_{\mathrm{C} 0} /$ $M_{\mathrm{C} 9} \geq 0.6$ have $R_{\text {disk,C} 0} \geq D(\mathrm{~L} 1-\mathrm{C} 0)$ and do not correspond to 
Table 2. Different models investigated to fit C6.

\begin{tabular}{lcc}
\hline \hline Model & Origin & $\chi^{2}\left(i_{\mathrm{o}}\right)$ \\
\hline Precession & C0 & Convex: No solution \\
BBH C0-C9 & C0 & Concave: Solution \\
BBH C0-C3 & C0 & Concave: Solution rejected \\
\hline
\end{tabular}

Table 3. Parameter ranges for the BBH system that ejects C6.

\begin{tabular}{ccc}
\hline \hline$V_{\mathrm{a}}$ & $0.001 c$ & $0.45 c$ \\
$T_{\mathrm{b}}\left(V_{\mathrm{a}}\right)$ & $\approx 6.2 \times 10^{5} \mathrm{yr}$ & $\approx 760 \mathrm{yr}$ \\
$\left(M_{\mathrm{C} 0}+M_{\mathrm{C} 9}\right)\left(V_{\mathrm{a}}\right)$ & $\approx 4.6 \times 10^{4} M_{\odot}$ & $\approx 3.1 \times 10^{10} M_{\odot}$ \\
\hline
\end{tabular}

stable solutions. Moreover, the solution with $M_{\mathrm{C} 0} / M_{\mathrm{C} 9} \approx 0.5$ has the smallest $\chi_{\min }^{2}$. Thus, component $\mathrm{C} 6$ is ejected from core $\mathrm{C} 0$ of the $\mathrm{BBH}$ system $\mathrm{C} 0-\mathrm{C} 9$, which is characterized by $R_{\mathrm{C} 0-\mathrm{C} 9} \approx 0.3$ mas and $M_{\mathrm{C} 0} / M_{\mathrm{C} 9} \approx 0.5$.

The corresponding solution is characterized by $\chi_{\min }^{2} \approx 36.9$, the inclination angle is $i_{\mathrm{o}} \approx 6.7^{\circ}$, the ratio $T_{\mathrm{p}} / T_{\mathrm{b}}$ is $\approx 5.5$, and the angle between the accretion disk and the rotation plane of the BBH system is $\Omega(\mathrm{C} 0) \approx 3.1^{\circ}$. The bulk Lorentz factor of the VLBI component is $\gamma_{\mathrm{c}} \approx 9.6$, and the mean apparent speed is $V_{\text {ap }}(\mathrm{C} 6) \approx 8 \mathrm{c}$. The ejection time of the VLBI component is $t_{\mathrm{o}} \approx 2002.4$.

Using the parameters of the solution, we gradually varied $V_{\mathrm{a}}$ between $0.001 c$ and $0.45 c$. The function $\chi^{2}\left(V_{\mathrm{a}}\right)$ remained constant, indicating a degeneracy of the solution (see Fig. B.6). We deduced the variation range of the $\mathrm{C} 0-\mathrm{C} 9$ parameters in the $\mathrm{BBH}$ system that eject C6. They are given in Table 3.

Based on the knowledge of the mass ratio $M_{\mathrm{C} 0} / M_{\mathrm{C} 9}$ and the ratio $T_{\mathrm{p}} / T_{\mathrm{b}}$, we calculated the mass of the ejecting $\mathrm{BH} M_{\mathrm{C} 0}$, the orbital period $T_{\mathrm{b}}$, and the precession period $T_{\mathrm{p}}$ for each value of $V_{\mathrm{a}}$. We found that the radius of the accretion disk around $\mathrm{C} 0$ does not depend on $V_{\mathrm{a}}$ and is $R_{\text {disk,C0 }}=0.106$ mas $=0.46 \mathrm{pc}$. The ratio $R_{\text {disk,C0 }} / R_{\mathrm{C} 0-\mathrm{L} 1}$ is $R_{\text {disk,C0}} / R_{\mathrm{C} 0-\mathrm{L} 1}=0.85$.

\subsection{Fit of component $\mathrm{C} 13$}

\subsubsection{Introduction}

We studied the four different cases given in Table 4 . We calculated the curve $\chi^{2}\left(i_{0}\right)$, which corresponds to the $\chi^{2}$ of the model of the VLBI component ejection for a given inclination angle $i_{\mathrm{o}}$. Details of the calculations corresponding to the four models are given in Appendix C. In this section, we present the results of fitting the ejection of component $\mathrm{C} 13$ from the origin $\mathrm{C} 9$ by the $\mathrm{BBH}$ system $\mathrm{C} 0-\mathrm{C} 9$.

\subsection{2. $\mathrm{BBH}$ system $\mathrm{C} 0-\mathrm{C} 9$ : Origin $\mathrm{C} 9$}

The BBH system is constituted by core $\mathrm{C} 0$ and the stationary component C9. Its separation is $R_{\mathrm{C} 0-\mathrm{C} 9}=0.3$ mas. Component $\mathrm{C} 13$ is ejected by the stationary component $\mathrm{C} 9$ (see Fig. 7).

We found that the mass ratio $M_{\mathrm{C} 9} / M_{\mathrm{C} 0}$ is $M_{\mathrm{C} 9} / M_{\mathrm{C} 0}>1$ (see Fig. C.5). Using the stability condition, $R_{\text {disk,C }}<D(\mathrm{~L} 1-\mathrm{C} 9)$, we found that solutions with the mass ratio $M_{\mathrm{C} 9} / M_{\mathrm{C} 0} \geq 3$ have $R_{\text {disk,C } 9} \geq D(\mathrm{~L} 1-\mathrm{C} 9)$ and do not correspond to stable solutions. Moreover, the solution with $M_{\mathrm{C} 9} / M_{\mathrm{C} 0}=2$ has the smallest $\chi_{\min }^{2}$. Thus, component $\mathrm{C} 13$ is ejected from the stationary component $\mathrm{C} 9$ of the $\mathrm{BBH}$ system $\mathrm{C} 0-\mathrm{C} 9$, which is
Table 4. Different models investigated for the fit of C13.

\begin{tabular}{ccc}
\hline \hline Model & Origin & $\chi^{2}\left(i_{\mathrm{o}}\right)$ \\
\hline Precession & C0 & Convex: No solution \\
Precession & C9 & Convex: No solution \\
BBH C0-C9 & C9 & Concave: Solution \\
BBH C0-C9 & C0 & Convex: No solution \\
\hline
\end{tabular}

Table 5. Parameter ranges for the BBH system that ejects $\mathrm{C} 13$.

\begin{tabular}{lcc}
\hline \hline$V_{\mathrm{a}}$ & $0.001 c$ & $0.45 c$ \\
$T_{\mathrm{b}}\left(V_{\mathrm{a}}\right)$ & $\approx 3.0 \times 10^{5} \mathrm{yr}$ & $\approx 360 \mathrm{yr}$ \\
$\left(M_{\mathrm{C} 0}+M_{\mathrm{C} 9}\right)\left(V_{\mathrm{a}}\right)$ & $\approx 2.0 \times 10^{5} M_{\odot}$ & $\approx 13.7 \times 10^{10} M_{\odot}$ \\
\hline
\end{tabular}

characterized by $R_{\mathrm{C} 0-\mathrm{C} 9}=0.3$ mas and $M_{\mathrm{C} 9} / M_{\mathrm{C} 0}=2 \pm 0.5$. The fit of component $\mathrm{C} 13$ ejected from the stationary component $\mathrm{C} 9$ provides the mass ratio inverse of the mass ratio provided by the fit of component $\mathrm{C} 6$ ejected from core $\mathrm{C} 0$. This showis that the $\mathrm{BBH}$ model is consistent.

The corresponding solution is characterized by $\chi_{\text {min }}^{2} \approx 9.3$, and the inclination angle is $i_{\mathrm{o}} \approx 5.8^{\circ}$. The ratio $T_{\mathrm{p}} / T_{\mathrm{b}}$ is $\approx 10.2$, and the angle between the accretion disk and the rotation plane of the BBH system is $\Omega(\mathrm{C} 9) \approx 3.3^{\circ}$. The bulk Lorentz factor of the VLBI component is $\gamma_{\mathrm{c}} \approx 7.3$, and the mean apparent speed is $V_{\text {ap }}(\mathrm{C} 6) \approx 6 c$. The ejection time of the VLBI component is $t_{\mathrm{o}} \approx 2011.6$.

Using the parameters of the solution, we gradually varied $V_{\mathrm{a}}$ between $0.001 c$ and $0.45 c$. The function $\chi^{2}\left(V_{\mathrm{a}}\right)$ remained constant, indicating a degeneracy of the solution (see Fig. C.8). We deduced the range of variation of the $\mathrm{BBH}$ system parameters ejecting $\mathrm{C} 13$. They are given in Table 5.

We found that component $\mathrm{C} 13$ cannot be ejected by a single spinning $\mathrm{BH}$ but must be ejected by a BBH system.

We found that the radius of the accretion disk around $\mathrm{C} 9$ does not depend on $V_{\mathrm{a}}$ and is $R_{\text {disk,C } 9}=0.140$ mas $=0.61 \mathrm{pc}$. The ratio $R_{\text {disk,C9}} / R_{\mathrm{C} 9-\mathrm{L} 1}$ is $R_{\mathrm{disk}, \mathrm{C} 9} / R_{\mathrm{C} 9-\mathrm{L} 1}=0.79$.

\subsection{Fit of component $\mathrm{C7}$}

\subsubsection{Introduction}

We studied the three different models given in Table 6. We calculated the curve $\chi^{2}\left(i_{0}\right)$, which corresponds to the $\chi^{2}$ of the model of the VLBI component ejection for a given inclination angle $i_{\mathrm{o}}$. The details of the calculations corresponding to the three models are given in Appendix D. In this section, we present the results of fitting the ejection of component $\mathrm{C} 7$ from the origin $\mathrm{C} 3$ by the $\mathrm{BBH}$ system $(\mathrm{C} 0+\mathrm{C} 9)-\mathrm{C} 3$.

We found that component $\mathrm{C} 7$ cannot be ejected by a single spinning BH but must be ejected by a BBH system.

\subsection{2. $\mathrm{BBH}$ system $(\mathrm{CO}+\mathrm{C} 9)-\mathrm{C} 3$ : Origin $\mathrm{C} 3$}

The $\mathrm{BBH}$ system is constituted by the system $\mathrm{C} 0+\mathrm{C} 9$ and the stationary component C3. Its separation is $R_{\mathrm{G}-\mathrm{C} 3}=0.37$ mas (see Fig. 1). Component $\mathrm{C} 7$ is ejected from the stationary component C3 (see Fig. 7).

We found that the mass ratio $M_{\mathrm{C} 3} /\left(M_{\mathrm{C} 0}+M_{\mathrm{C} 9}\right)$ is $M_{\mathrm{C} 3} /\left(M_{\mathrm{C} 0}+M_{\mathrm{C} 9}\right) \leq 0.1$ (see Fig. D.1). We found that solutions 
Table 6. Different models investigated for the fit of C7.

\begin{tabular}{lcc}
\hline \hline Model & Origin & $\chi^{2}\left(i_{\mathrm{o}}\right)$ \\
\hline Precession & C0 & Convex: No solution \\
BBH (C0+C9)-C3 & C3 & Concave: Solution \\
BBH C0-C9 & C0 & Concave: Solution rejected \\
\hline
\end{tabular}

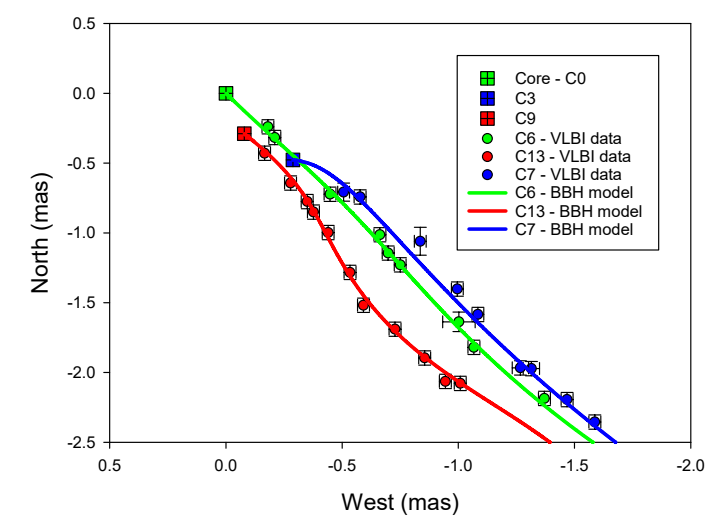

Fig. 7. Nucleus of $2201+315$ with its three BHs. They are associated with the core, i.e., component $\mathrm{C} 0$, and the stationary components $\mathrm{C} 9$ and $\mathrm{C} 3$. The VLBI components C6, C13, and C7 are ejected from the $\mathrm{BHs} \mathrm{C} 0, \mathrm{C} 9$, and $\mathrm{C} 3$, respectively. We show the component positions provided by Lister et al. (2019) and the trajectories calculated using the BBH model.

with the mass ratio $M_{\mathrm{C} 3} /\left(M_{\mathrm{C} 0}+M_{\mathrm{C} 9}\right)<0.05$ have a minimum, but solutions with $M_{\mathrm{C} 3} /\left(M_{\mathrm{C} 0}+M_{\mathrm{C} 9}\right) \geq 0.05$ do not have a minimum because when $i_{\mathrm{o}}$ becomes smaller than about $4.8^{\circ}$, the radius of the accretion disk diverges and becomes larger than the $\mathrm{BBH}$ system $R_{\mathrm{G}-\mathrm{C} 3}$ (see Fig. D.2). We adopted the solution with $M_{\mathrm{C} 3} /\left(M_{\mathrm{C} 0}+M_{\mathrm{C} 9}\right) \approx 0.01$, which corresponds to the best solution. Thus, component $\mathrm{C} 7$ is ejected from the stationary component $\mathrm{C} 3$ of the $\mathrm{BBH}$ system $(\mathrm{C} 0+\mathrm{C} 9)-\mathrm{C} 3$, which is characterized by $R_{\mathrm{G}-\mathrm{C} 3}=0.37$ mas and $M_{\mathrm{C} 3} /\left(M_{\mathrm{C} 0}+M_{\mathrm{C} 9}\right) \approx 0.01$.

The corresponding solution is characterized by $\chi_{\min }^{2} \approx 38.4$, and the inclination angle is $i_{\mathrm{o}} \approx 6.9^{\circ}$. The ratio $T_{\mathrm{p}} / T_{\mathrm{b}}$ is $\approx 10.7$, and the angle between the accretion disk and the rotation plane of the BBH system is $\Omega(\mathrm{C} 0) \approx 3.8^{\circ}$. The bulk Lorentz factor of the VLBI component is $\gamma_{\mathrm{c}} \approx 7.1$, and the mean apparent speed is $V_{\text {ap }}(\mathrm{C} 7) \approx 6 c$. The ejection time of the VLBI component is $t_{\mathrm{o}} \approx 2005.4$.

Using the parameters of the solution, we gradually varied $V_{\mathrm{a}}$ between $0.001 c$ and $0.45 c$. The function $\chi^{2}\left(V_{\mathrm{a}}\right)$ remained constant, indicating a degeneracy of the solution (see Fig. D.3). We deduced the parameter range of variation of the BBH system that ejects C7. They are given in Table 7.

We found that the radius of the accretion disk around $\mathrm{C} 3$ does not depend on $V_{\mathrm{a}}$ and is $R_{\text {disk,C3 }}=0.020$ mas $=0.09 \mathrm{pc}$. The ratio $R_{\text {disk,C3}} / R_{\mathrm{C} 3-\mathrm{L} 1^{\prime}}$ is $R_{\mathrm{disk}, \mathrm{C} 3} / R_{\mathrm{C} 3-\mathrm{L} 1^{\prime}}=0.59$, where $L 1^{\prime}$ is the first Lagrange point of the system $(\mathrm{C} 0+\mathrm{C} 9)-\mathrm{C} 3$.

\section{7. $\mathrm{BH}$ system characteristics in $2201+315$}

The inclination angle of $2201+315$ is $5.8^{\circ} \leq i_{\mathrm{o}} \leq 6.9^{\circ}$. The kiloparsec-scale radio map (Cooper et al. 2007) shows two extended lobes, and the total extension of the source is about 75 arcsec. When we assume $i_{\mathrm{o}} \approx 6.5^{\circ}$, the total extension of the extended lobes is about $2.9 \mathrm{Mpc}$. The structure of the nucleus is shown in Fig. 1.
Table 7. Parameter ranges for the BBH system that ejects C7.

\begin{tabular}{lcc}
\hline \hline$V_{\mathrm{a}}$ & $0.001 c$ & $0.45 c$ \\
$T_{\mathrm{b}}\left(V_{\mathrm{a}}\right)$ & $\approx 9.2 \times 10^{5} \mathrm{yr}$ & $\approx 1100 \mathrm{yr}$ \\
$\left(M_{\mathrm{C} 0+\mathrm{C} 9}+M_{\mathrm{C} 3}\right)\left(V_{\mathrm{a}}\right)$ & $\approx 4.0 \times 10^{4} M_{\odot}$ & $\approx 2.8 \times 10^{10} M_{\odot}$ \\
\hline
\end{tabular}

Table 8. Characteristics of the $\mathrm{BH}$ system, whose components are $\mathrm{C} 9$, $\mathrm{C} 0$ (core), and $\mathrm{C} 3$.

\begin{tabular}{|c|}
\hline 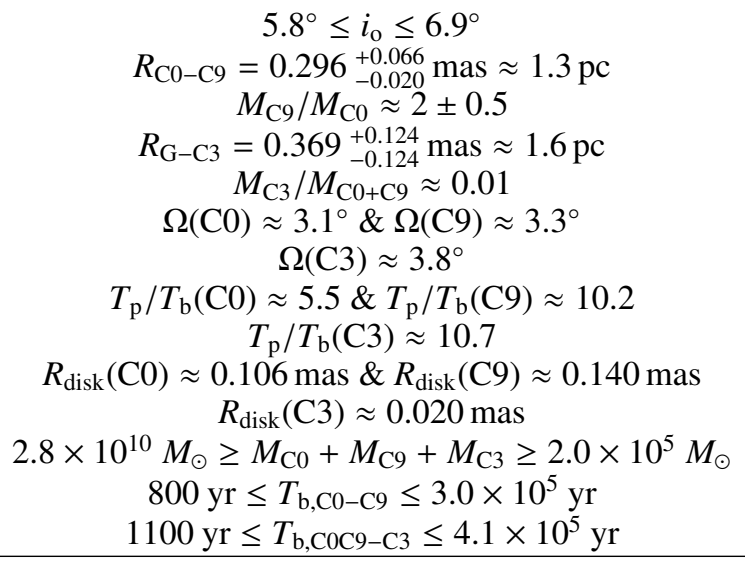 \\
\hline
\end{tabular}

To obtain the final range of the parameters of the $\mathrm{BH}$ system $\mathrm{C} 9-\mathrm{C} 0-\mathrm{C} 3$, we intersected the ranges of the $\mathrm{BBH}$ system parameters we found after the fits of $\mathrm{C} 13, \mathrm{C} 6$, and C7. The characteristics of the $\mathrm{BH}$ system $\mathrm{C} 9-\mathrm{C} 0-\mathrm{C} 3$ are given in Table 8 where $\Omega$ is the angle between the accretion disk and the rotation plane of the BHs.

The $\mathrm{BH}$ system and the component trajectories of $\mathrm{C} 6, \mathrm{C} 7$, and $\mathrm{C} 13$ using the BBH model we obtained in Sects. 3.4, 3.6, and 3.5 are shown in Fig. 7.

\section{Discussion and conclusion}

The analysis of MOJAVE model fit data with our minimization method suggests that none of components C6, C7, and C13 can be ejected by a single spinning $\mathrm{BH}$, but that they are ejected by BBH systems and that the nucleus of $2201+315$ contains three BHs. The three BHs are associated with the VLBI core $\mathrm{C} 0$ and the stationary components C9 and C3 as defined by Lister et al. (2019). This source constitutes another example of a nucleus that contains several BHs that are detected in radio and eject VLBI components.

Stationary VLBI components are frequently observed (Jorstad et al. 2017) and are generally assumed to be associated with stationary or recollimation shocks (see Mizuno et al. 2015, Martí et al. 2016, Hada et al. 2018 and references therein), but in the two-fluid model when the relativistic $\mathrm{e}^{-}-\mathrm{e}^{+}$beam dissipates into the subrelativistic $\mathrm{e}^{-}-\mathrm{p}^{+}$jet, a VLBI component is observed that moves subrelativistically. This appears as a quasi stationary VLBI component (see, e.g., component 1 of 1532+016; Lister et al. 2019), and finally the stationary components can also be associated with BHs that eject VLBI components. The stationary components are not necessarily all associated with BHs or with recollimation shocks. To explain the MOJAVE observations of $2201+315$, we do not need stationary or recollimation shocks.

As indicated in Roland et al. (2015), a BBH system can produce three perturbations of the VLBI ejection by the precession 


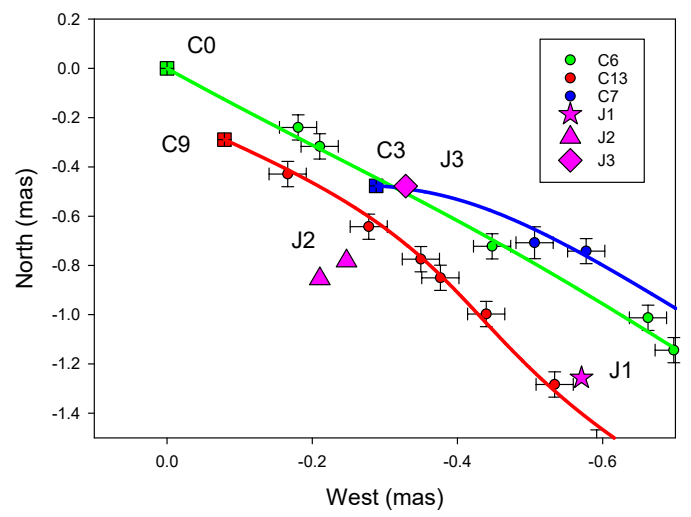

Fig. 8. Positions of components $\mathrm{J} 1, \mathrm{~J} 2$, and $\mathrm{J} 3$ detected at 43 and $86 \mathrm{GHz}$ by Cheng et al. (2018). The VLBI components C6, C13, and C7 are shown.

of the accretion disk, the motion of the two BHs around the center of gravity of the BBH system, and the possible motion of the $\mathrm{BBH}$ system around either a third $\mathrm{BH}$ or another BBH system. This third perturbation produces a change in the VLBI jet direction of between 5 to 15 mas. It is observed for instance in the cases of 1928+738 (Roland et al. 2015), 3C 345 (Lister et al. 2019), BL Lac (Lister et al. 2019), and 3C 454.3 (Lister et al. 2019).

When we observe a change in VLBI jet direction between 5 to 15 mas, for example, the slow rotation of the BBH system, has to be modeled and the VLBI data have then to be corrected for this slow rotation, so that finally the ejection of the corrected data can be modeled using a BBH system. This has been done by Roland et al. (2015) in the case of $1928+738$, which contains two BBH systems. In the case of $2201+315$, the mass of the third BH is far lower than that of the two others, and the ejection of components $\mathrm{C} 6$ and $\mathrm{C} 13$ can be modeled using the $\mathrm{BBH}$ system $\mathrm{C} 0-\mathrm{C} 9$ and the ejection of component $\mathrm{C} 7$ using the $\mathrm{BBH}$ system $(\mathrm{C} 0+\mathrm{C} 9)-\mathrm{C} 3$.

As indicated in the introduction, when the nucleus contains a BBH system, the two BHs can eject VLBI components and both can be detected using VLBI observations, but it is not necessarily always the case. Roland et al. (2015) showed that $1928+738$ contains two BBH systems: the two BHs of the first $\mathrm{BBH}$ system are detected (associated with a stationary component) and eject VLBI components; only one BH of the second $\mathrm{BBH}$ system ejects VLBI components during the observations; no $\mathrm{BH}$ of the second $\mathrm{BBH}$ system is associated with a stationary component.

The source $2201+315$ has been observed between 1995 and 2018 in the MOJAVE survey. While core $\mathrm{C} 0$ is detected during the whole period, component C3 is detected between 1995 and 2010 and component C9 is detected between 2009 and 2017. Because the stationary components $\mathrm{C} 3$ and $\mathrm{C} 9$ correspond the place in the VLBI jet that becomes transparent to a given synchrotron frequency (see Sect. 3.2), the disappearance of component C3 in 2013 means that at least the ejection of the relativistic $\mathrm{e}^{-} \mathrm{e}^{+}$plasma (the beam) stopped in 2010; this does not mean that the ejection of the subrelativistic $\mathrm{e}^{-} \mathrm{p}$ plasma (the jet) also stopped in 2010. Component C9 started to be detected in 2009, which means that the ejection of the relativistic $\mathrm{e}^{-} \mathrm{e}^{+}$plasma (the beam) started in 2010.

Source $2201+315$ has been observed at 43 and $86 \mathrm{GHz}$ by Cheng et al. (2018). They detected three components J1, J2, and J3. Their positions are shown in Fig. 8. Component J1 probably belongs to the family of trajectories defined by $\mathrm{C} 13$ and has been ejected from the stationary component C9. It is just as probable
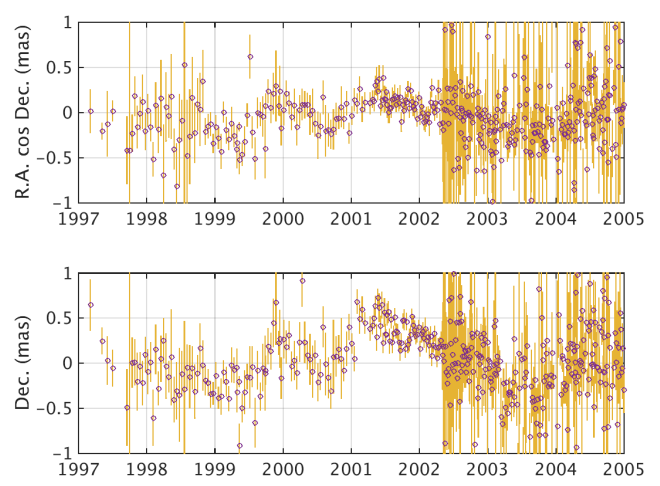

Fig. 9. Coordinate time series of $2201+315$ (here restricted to $1997-$ 2005) obtained after the analysis of geodetic VLBI data. The position is given as offsets on the local plane of the sky from the average position of the source on the whole observation period of geodetic VLBI (1979-2018). Dots and gray bars show the estimated positions and their uncertainties for each of these sessions. The weighted rms are 0.24 mas in $\alpha \cos \delta$ and 0.34 mas in $\delta$.

that component $\mathrm{J} 3$ belongs to the family of trajectories defined by $\mathrm{C} 6$ and has been ejected from core $\mathrm{C} 0$ or is ejected from the stationary component $\mathrm{C} 3$. For component $\mathrm{J} 2$, the situation is less clear because of the shift that is observed with the family of trajectories defined by $\mathrm{C} 13$, but it can probably be associated with the family of trajectories defined by $\mathrm{C} 13$ and has been ejected from the stationary component $\mathrm{C} 9$.

An interesting corollary of our study resides in the importance of detecting and separating possible stationary components and determining their flux density ratios, and thereby explaining the position variations measured during geodetic VLBI sessions. Radio source $2201+315$ has been intensively observed in the framework of the permanent geodetic VLBI program devoted to absolute astrometry (Fey et al. 2015). In geodetic VLBI analysis, the coordinates of the "radio center" can be estimated on average over observing sessions of $24 \mathrm{~h}$ each, and session-wise coordinate time series can be obtained in this way. The radio center is not located at the brightest component of the radio source but can rather be seen as a barycenter of the brightness distribution mitigated by the effect of the network geometry. The accuracy of coordinates as determined by geodetic VLBI is typically a few dozen microarcseconds. This allows recording signatures of subtle changes in the source structure as small variations in the coordinates. Radio source $2201+315$ was observed in more than 1000 sessions since 1979, mainly between 1990 and 2010. In Fig. 9 each dot represents the position of the radio center determined in one session of $24 \mathrm{~h}$ (see Gattano et al. 2018, for more details). During some periods, dramatic changes in the coordinate time series can be observed. These variations can be explained by two types of variability that occur in the nucleus, that is, the ejection of a new VLBI component whose flux density can be higher than the flux densities of the two stationary components, and in the case of a $\mathrm{BBH}$, the variations in the ratio of the flux densities of the VLBI components associated with two BHs.

It is generally observed that changes in astrometric position correspond to the direction of the VLBI jet (Moór et al. 2011). However, if the direction of the BBH system is different from the direction of the VLBI jet, these two types of variations will produce changes in astrometric position in two different directions. During 1997-2005, only components C0 and C3 of the three stationary components were detected. The flux densities of the two component change with time. Generally, the flux density 


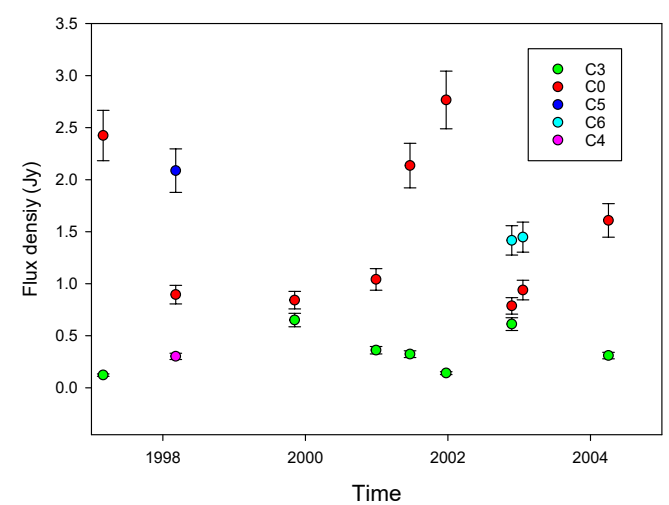

Fig. 10. Flux densites of core $\mathrm{C} 0$, the second $\mathrm{BH}, \mathrm{C} 3$, and the flux densities of components $\mathrm{C} 4, \mathrm{C} 5$, and $\mathrm{C} 6$ when they were close to the $\mathrm{BBH}$ system during the period 1997-2005. The flux densities are taken from Lister et al. (2019).

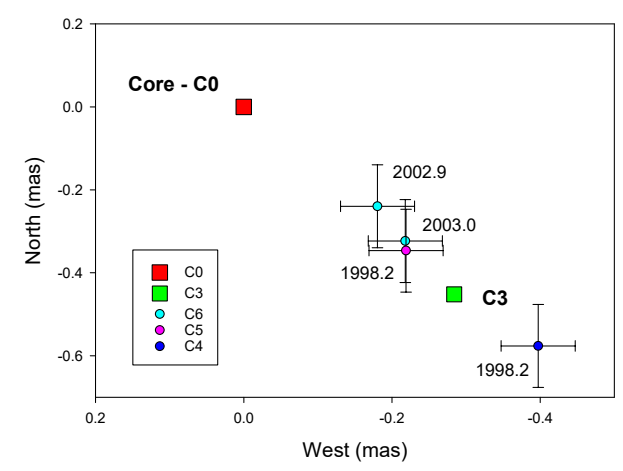

Fig. 11. Positions of components C4, C5, and C6 in 1998.2 and $\approx 2003$. The VLBI coordinates are taken from Lister et al. (2019).

of the core, $S_{v}(\mathrm{C} 0)$, is higher than the flux density of component C3, $S_{v}(\mathrm{C} 3)$. However, the flux densities of the core and C3 can change rapidly and significantly, and consequently, there are periods where the ratio $S_{v}(\mathrm{C} 0) / \mathrm{S}_{v}(\mathrm{C} 3)$ can be $S_{v}(\mathrm{C} 0) / \mathrm{S}_{v}(\mathrm{C} 3) \approx$ 1. In 1998-1999, the flux density of the core was similar to the flux density of $\mathrm{C} 3$, and the flux density of the nucleus was dominated by the flux density of component $\mathrm{C} 5$, which was close to $\mathrm{C} 3$ (see Figs. 10 and 11). Thus the mean astrometric position deduced at $8 \mathrm{GHz}$ is shifted to the south. Between 2001 and 2002, the flux density of the nucleus was dominated by the flux density of the core, $\mathrm{C} 0$, which increased with time, and the mean astrometric position deduced at $8 \mathrm{GHz}$ is shifted to the north. However, in 2003, the flux density of the core, $\mathrm{C}$, was similar to the flux density of $\mathrm{C} 3$ and the flux density of the nucleus was dominated by the flux density of component C6, which was close to $\mathrm{C} 3$ (see Figs. 10 and 11). Thus the mean position deduced at $8 \mathrm{GHz}$ is shifted to the south and we observe a quick change in the astrometric position time series. During the periods 2009 to 2012 and 2014 to 2015, the flux density of component C9 was higher than the flux density of the core (see Fig. 12). During these periods, the astrometric position is shifted along the direction $\mathrm{C} 0-\mathrm{C}$, which is different from the VLBI jet direction. Thus the astrometric position of $2201+315$ moves with time in two different directions. The mean astrometric position of $2201+315$ is located between the stationary components $\mathrm{C} 0, \mathrm{C} 9$, and $\mathrm{C} 3$ and does not correspond to any of their positions.

This result shows the importance of determining the structure of nuclei of extragalactic radio sources and especially of quasars

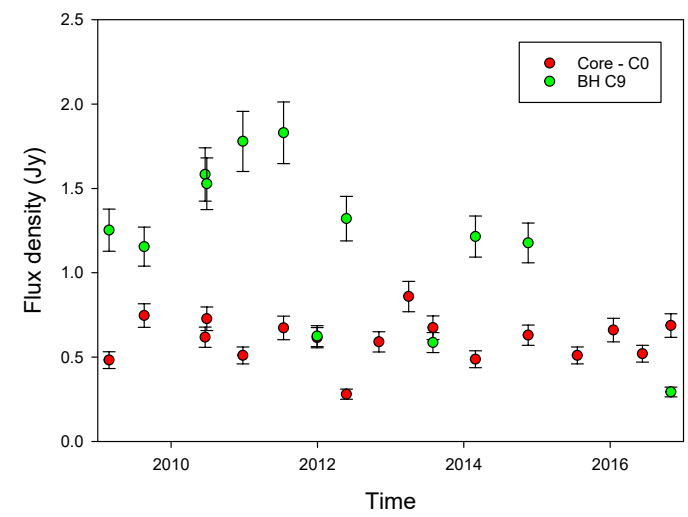

Fig. 12. Flux densities of the core and component C9. The flux density of component $\mathrm{C} 9$ is higher than the flux density of the core during the periods 2009 to 2012 and 2014 to 2015 . The flux densites are taken from Lister et al. (2019).

using VLBI observations at frequencies $\geq 15 \mathrm{GHz}$ in order to detect and separate possible stationary components and to determine their flux density ratio. This will allow explaining the time shifts of the position observed during geodetic VLBI observations and Gaia observations. This also shows the importance of large MOJAVE-like VLBI databases for improving the understanding of the apparent displacement of the radio center measured by geodetic VLBI and thereby in the maintenance of the celestial reference frames.

Acknowledgements. We thank Prof. Lister who provided us with the new MOJAVE data before publication. We are also grateful to an anonymous referee whose comments helped in improving this work. This research has made use of data from the MOJAVE database that is maintained by the MOJAVE team (Lister et al. 2019).

\section{References}

Acero, F., Ackermann, M., Ajello, M., et al. 2015, ApJS, 218, 23 Begelman, M. C., Blandford, R. D., \& Rees, M. J. 1980, Nature, 287, 307 Britzen, S., Roland, J., Laskar, J., et al. 2001, A\&A, 374, 784 Brown, A. G. A., Vallenari, A., Prusti, T., et al. 2018, A\&A, 616, A1 Carrasco, J. M., Evans, D. W., Montegriffo, P., et al. 2016, A\&A, 595, A7 Cheng, X. P., An, T., Hong, X. Y., et al. 2018, ApJS, 234, 17

Colpi, M. 2014, Space Sci. Rev., 183, 189

Colpi, M., \& Dotti, M. 2011, Adv. Sci. Lett., 4, 181

Cooper, N. J., Lister, M. L., \& Kochanczyk, M. D. 2007, ApJS, 171, 376

Fey, A. L., Gordon, D., Jacobs, C. S., et al. 2015, ApJS, 150, 58

Gattano, C., Lambert, S. B., \& Le Bail, K. 2018, A\&A, 618, A80

Hada, K., Doi, A., Wajima, K., et al. 2018, ApJ, 860, 141

Healey, S. E., Romani, R. W., Cotter, G., et al. 2008, ApJS, 175, 97

Hewitt, A., \& Burbidge, G. 1993, ApJS, 87, 451

Ho, L. C., \& Kim, M. 2009, ApJS, 184, 398

Hutchings, J. B., \& Neff, S. G. 1992, ApJS, 104, 1

Hutchings, J. B., \& Morris, S. C. 1995, ApJS, 109, 1541

Jorstad, S. G., Marscher, A. P., Morozova, D. A., et al. 2017, ApJ, 846, 98

Katz, J. I. 1997, ApJ, 478, 527

Lister, M. L., Homan, D. C., Hovatta, T., et al. 2019, ApJ, 874, 43

Lobanov, A. P., \& Roland, J. 2005, A\&A, 431, 831

Marscher, A. P., Jorstad, S. G., D'Arcangelo, F. D., et al. 2008, Nature, 452, 966

Martí, J. M., Perucho, M., \& Gómez, J. L. 2016, ApJ, 831, 163

Maselli, A., Cusumano, G., Massaro, E., et al. 2010, A\&A, 520, A47

Mizuno, Y., Gómez, J. L., Nishikawa, K.-I., et al. 2015, ApJ, 809, 38

Moór, A., Frey, S., Lambert, S. B., Titov, O. A., \& Bakos, J. 2011, ApJS, 141, 178

Roland, J., Britzen, S., Kudryavtseva, N. A., Witzel, A., \& Karouzos, M. 2008, A\&A, 483, 125

Roland, J., Britzen, S., Caproni, A., et al. 2013, A\&A, 557, A85

Roland, J., Britzen, S., Kun, E., et al. 2015, A\&A, 578, A86

Volonteri, M., Bogdanović, T., Dotti, M., \& Colpi, M. 2016, IAU Focus Meeting, 29,285 


\section{Appendix A: Model to fit VLBI observations}

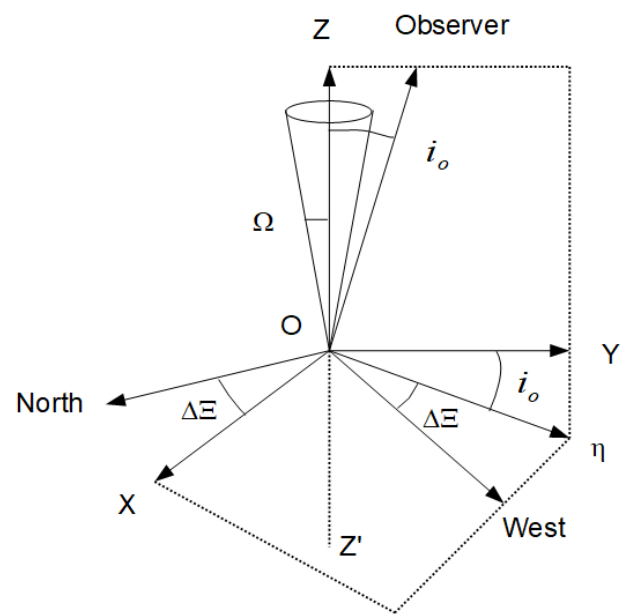

Fig. A.1. Geometry of the problem. The planes $X-\eta$ and west-north are perpendicular to the line of sight. In the west-north plane, the axis $\eta$ corresponds to the mean ejection direction of the VLBI component. $\Omega$ is the opening angle of the precession cone.

Before fitting components $\mathrm{C} 6, \mathrm{C} 7$, and $\mathrm{C} 13$, we recall the geometry, parameters, and basic equations of the model, which have been developed in Britzen et al. (2001), Lobanov \& Roland (2005), and Roland et al. (2008, 2013). Details can be found in Roland et al. (2008, 2013).

\section{A.1. Geometry of the model}

We call $\Omega$ the angle between the accretion disk and the orbital plane $(X O Y)$ of the $\mathrm{BBH}$ system. The component is ejected in a cone (the precession cone) with its axis in the $Z^{\prime} O Z$ plane and its opening angle is $\Omega$. We assumed that the line of sight is in the plane (YOZ) and that it forms an angle $i_{\mathrm{o}}$ with the axis $Z^{\prime} O Z$ (see Fig. A.1). The axis $\eta$ corresponds to the mean ejection direction of the VLBI component projected on a plane perpendicular to the line of sight, so that the plane perpendicular to the line of sight is the plane $(\eta O X)$. We call $\Delta \Xi$ the rotation angle in the plane perpendicular to the line of sight to transform the coordinates $\eta$ and $X$ into coordinates $N$ (north) and $W$ (west), which are directly comparable with the VLBI observations. We have

$W=-x \cos (\Delta \Xi)+\left(z \sin \left(i_{\mathrm{o}}\right)+y \cos \left(i_{\mathrm{o}}\right)\right) \sin (\Delta \Xi)$,

$N=x \sin (\Delta \Xi)+\left(z \sin \left(i_{0}\right)+y \cos \left(i_{0}\right)\right) \cos (\Delta \Xi)$.

The sign of the coordinate $\mathrm{W}$ was changed from Roland et al. (2008) to use the same definition as in the VLBI observations.

\section{A.2. General perturbation of the VLBI ejection}

For VLBI observations, the origin of the coordinates is $\mathrm{BH} 1$, that is, the $\mathrm{BH}$ that ejects the VLBI components. For the sake of simplicity, we assumed that the two BHs have circular orbits, that is, $e=0$. Therefore, the coordinates of the moving components in the frame of reference where $\mathrm{BH} 1$ is considered the ejection origin are (Roland et al. 2008)

$$
\begin{aligned}
x_{\mathrm{c}}= & {\left[R_{\mathrm{o}}(z) \cos \left(\omega_{\mathrm{p}} t-k_{\mathrm{p}} z(t)+\phi_{\mathrm{o}}\right)\right.} \\
& \left.+x_{1} \cos \left(\omega_{\mathrm{b}} t-k_{\mathrm{b}} z(t)+\psi_{\mathrm{o}}\right)-x_{1} \cos \left(\psi_{\mathrm{o}}\right)\right] \\
& \exp \left(-t / T_{\mathrm{d}}\right),
\end{aligned}
$$

$$
\begin{aligned}
y_{\mathrm{c}}= & {\left[R_{\mathrm{o}}(z) \sin \left(\omega_{\mathrm{p}} t-k_{\mathrm{p}} z(t)+\phi_{\mathrm{o}}\right)\right.} \\
& \left.+y_{1} \sin \left(\omega_{\mathrm{b}} t-k_{\mathrm{b}} z(t)+\psi_{\mathrm{o}}\right)-y_{1} \sin \left(\psi_{\mathrm{o}}\right)\right] \\
& \exp \left(-t / T_{\mathrm{d}}\right), \\
z_{\mathrm{c}}= & z_{\mathrm{c}}(t),
\end{aligned}
$$

where $R_{\mathrm{o}}(z)$ is the amplitude of the precession perturbation, given by $R_{\mathrm{o}}(z)=R_{\mathrm{o}} z_{\mathrm{c}}(t) /\left(a+z_{\mathrm{c}}(t)\right)$, with $a=R_{\mathrm{o}} /(2 \tan \Omega) ; \omega_{\mathrm{p}}$ is $\omega_{\mathrm{p}}=2 \pi / T_{\mathrm{p}}$, where $T_{\mathrm{p}}$ is the precession period, and $k_{\mathrm{p}}$ is defined by $k_{\mathrm{p}}=2 \pi / T_{\mathrm{p}} V_{\mathrm{a}}$, where $V_{\mathrm{a}}$ is the speed of the propagation of the perturbations; $\omega_{\mathrm{b}}$ is $\omega_{\mathrm{b}}=2 \pi / T_{\mathrm{b}}$, where $T_{\mathrm{b}}$ is the BBH system period and $k_{\mathrm{b}}$ is defined by $k_{\mathrm{b}}=2 \pi / T_{\mathrm{b}} V_{\mathrm{a}} ; T_{\mathrm{d}}$ is the characteristic time of the damping of the perturbation, and $x_{1}$ and $y_{1}$ are given by

$$
x_{1}=y_{1}=-\frac{M_{2}}{M_{1}+M_{2}} \times\left[\frac{T_{\mathrm{b}}^{2}}{4 \pi^{2}} G\left(M_{1}+M_{2}\right)\right]^{1 / 3} \text {. }
$$

We define with $R_{\text {bin }}$ the distance between the two BHs as the separation of the $\mathrm{BBH}$ system. It is

$$
R_{\text {bin }}=\left[\frac{T_{\mathrm{b}}^{2}}{4 \pi^{2}} G\left(M_{1}+M_{2}\right)\right]^{1 / 3} .
$$

The differential equation governing the evolution of $z_{\mathrm{c}}(t)$ can be obtained by defining the speed of the component,

$v_{\mathrm{c}}^{2}=\left(\frac{\mathrm{d} x_{\mathrm{c}}(t)}{\mathrm{d} t}\right)^{2}+\left(\frac{\mathrm{d} y_{\mathrm{c}}(t)}{\mathrm{d} t}\right)^{2}+\left(\frac{\mathrm{d} z_{\mathrm{c}}(t)}{\mathrm{d} t}\right)^{2}$,

where $v_{\mathrm{c}}$ is related to the bulk Lorentz factor by $v_{\mathrm{c}} / c=$ $\sqrt{\left(1-1 / \gamma_{\mathrm{c}}^{2}\right)}$.

Using Eqs. (A.3), (A.4), and (A.5), we find from Eq. (A.8) that $\mathrm{d} z_{\mathrm{c}} / \mathrm{d} t$ is the solution of equation

$A\left(\frac{\mathrm{d} z_{\mathrm{c}}}{\mathrm{d} t}\right)^{2}+B\left(\frac{\mathrm{d} z_{\mathrm{c}}}{\mathrm{d} t}\right)+C=0$

The coefficients $A, B$, and $C$ are calculated in Appendix A of Roland et al. (2008). Equation (A.9) admits two solutions corresponding to the jet and the counter-jet.

\section{A.3. Coordinates of the VLBI component}

Solving Eq. (A.9), we determine the coordinate $z_{\mathrm{c}}(t)$ of a pointsource component that is ejected relativistically in the perturbed beam. Then, using Eqs. (A.3) and (A.4), we can find the coordinates $x_{\mathrm{c}}(t)$ and $y_{\mathrm{c}}(t)$ of the component. In addition, for each point of the trajectory, we can calculate the derivatives $\mathrm{d} x_{\mathrm{c}} / \mathrm{d} t, \mathrm{~d} y_{\mathrm{c}} / \mathrm{d} t$, and $\mathrm{d} z_{\mathrm{c}} / \mathrm{d} t$ and then deduce $\cos \theta, \delta_{\mathrm{c}}, S_{v}$, and $t_{\mathrm{obs}}$ (see Roland et al. 2013).

After calculating the coordinates $x_{\mathrm{c}}(t), y_{\mathrm{c}}(t)$, and $z_{\mathrm{c}}(t)$, they can be transformed into the $w_{\mathrm{c}}(t)$ (west) and $n_{\mathrm{c}}(t)$ (north) coordinates using Eqs. (A.1) and (A.2).

As explained in Britzen et al. (2001), Lobanov \& Roland (2005), and Roland et al. (2008), the radio VLBI component has to be described as an extended component along the beam. We call $n_{\text {rad }}$ the number of points (or integration steps along the beam) for which we integrate to model the component. The coordinates $W_{\mathrm{c}}(t)$ and $N_{\mathrm{c}}(t)$ of the VLBI component are then

$W_{\mathrm{c}}(t)=\left(\sum_{i=1}^{n_{\mathrm{rad}}} w_{\mathrm{c} i}(t)\right) / n_{\mathrm{rad}}$ 
and

$N_{\mathrm{c}}(t)=\left(\sum_{i=1}^{n_{\mathrm{rad}}} n_{\mathrm{c} i}(t)\right) / n_{\mathrm{rad}}$

and can be compared with the observed coordinates of the VLBI component, which correspond to the radio peak intensity coordinates provided by model-fitting during the VLBI data reduction process.

\section{A.4. Model parameters}

In this section, we list the possible free model parameters. They are: $i_{\mathrm{O}}$ the inclination angle; $\phi_{\mathrm{o}}$ the phase of the precession at $t=0 ; \Delta \Xi$ the rotation angle in the plane perpendicular to the line of sight (see Eqs. (A.1) and (A.2)); $\Omega$ the opening angle of the precession cone; $R_{\mathrm{O}}$ the maximum amplitude of the perturbation; $T_{\mathrm{p}}$ the precession period of the accretion disk; $T_{\mathrm{d}}$ the characteristic time for the damping of the beam perturbation; $M_{1}$ the mass of the $\mathrm{BH}$ ejecting the radio jet; $M_{2}$ the mass of the secondary $\mathrm{BH} ; \gamma_{\mathrm{c}}$ the bulk Lorentz factor of the VLBI component; $\psi_{\mathrm{o}}$ the phase of the BBH system at $t=0 ; T_{\mathrm{b}}$ the period of the $\mathrm{BBH}$ system; $t_{\mathrm{o}}$ the time at which the VLBI component is ejected; $V_{\mathrm{a}}$ the propagation speed of the perturbations; $n_{\text {rad }}$ the number of steps to describe the extension of the VLBI component along the beam; $\Delta W$ and $\Delta N$ the possible offsets of the origin of the VLBI component.

The parameter $n_{\text {rad }}$ is known when the size of the VLBI component is known. In this article we fit components C6, C7, and C13 assuming that their projected size on the sky is $=0.1$ mas. This means that practically, the problem we have to solve is a 15 free-parameter problem.

We have to investigate the different possible scenarios with regard to the sense of the rotation of the accretion disk and the sense of the orbital rotation of the BBH system. These possibilities correspond to $\pm \omega_{\mathrm{p}}\left(t-z / V_{\mathrm{a}}\right)$ and $\pm \omega_{\mathrm{b}}\left(t-z / V_{\mathrm{a}}\right)$. Because the sense of the precession is always opposite to the sense of the orbital motion (Katz 1997), we study the two cases denoted by +- and -+ , where we have $\omega_{\mathrm{p}}\left(t-z / V_{\mathrm{a}}\right),-\omega_{\mathrm{b}}\left(t-z / V_{\mathrm{a}}\right)$ and $-\omega_{\mathrm{p}}\left(t-z / V_{\mathrm{a}}\right), \omega_{\mathrm{b}}\left(t-z / V_{\mathrm{a}}\right)$, respectively.

\section{A.5. Method for solving the problem}

This method is a practical one that provides solutions, but the method is not unique and does not guarantee that all possible solutions are found. We calculate the projected trajectory on the plane of the sky of an ejected component and determine the parameters of the model to simultaneously produce the best fit with the observed west and north coordinates. The parameters we found minimize

$\chi_{\mathrm{t}}^{2}=\chi^{2}\left(W_{\mathrm{c}}(t)\right)+\chi^{2}\left(N_{\mathrm{c}}(t)\right)$,

where $\chi^{2}\left(W_{\mathrm{c}}(t)\right)$ and $\chi^{2}\left(N_{\mathrm{c}}(t)\right)$ are the $\chi^{2}$ calculated by comparing the VLBI observations with the calculated coordinates $W_{\mathrm{c}}(t)$ and $N_{\mathrm{c}}(t)$ of the component. For instance, to determine the inclination angle that provides the best fit, we minimize $\chi_{\mathrm{t}}^{2}\left(i_{\mathrm{o}}\right)$.

The concave parts of the surface $\chi^{2}\left(i_{0}\right)$ contain a minimum. We can find solutions without a minimum; they correspond to the convex parts of the surface $\chi^{2}\left(i_{0}\right)$ and are called mirage solutions.

To illustrate the properties of the surface $\chi^{2}\left(i_{0}\right)$, we plot in Fig. A.2 a possible example of a profile of the solution $\chi^{2}\left(i_{0}\right)$. Figure A. 2 shows two possible solutions for which $\chi^{2}$ (Sol1) $\approx$ $\chi^{2}$ (Sol2). Solution 2 is more robust than solution 1 , that is, it is the deepest one, and we adopted this solution.

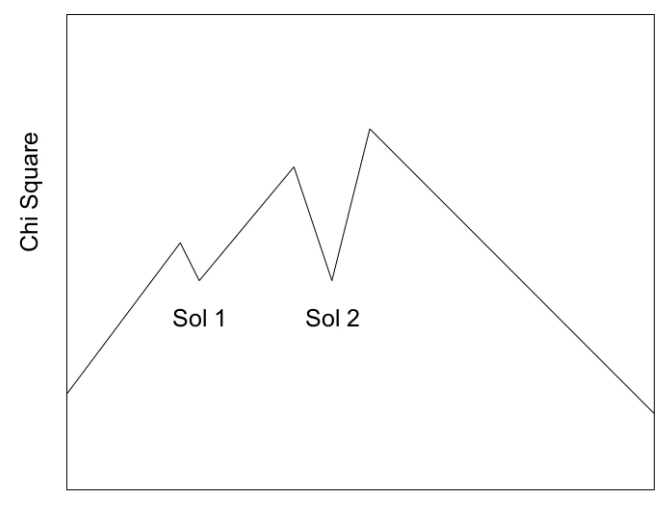

Inclination

Fig. A.2. Example of a possible profile of the solution $\chi^{2}\left(i_{0}\right)$. There are two possible solutions for which $\chi^{2}(\operatorname{Sol} 1) \approx \chi^{2}(\operatorname{Sol} 2)$. They correspond to the concave parts of the surface $\chi^{2}\left(i_{0}\right)$. However, solution 2 is more robust than solution 1, i.e., it is the deepest one, and we adopted this solution.

We define the robustness of the solution as the square root of the difference between the smallest maximum close to the minimum and the minimum of the function $\chi^{2}$. A solution of robustness 3 is a $3 \sigma$ solution, that is, $3 \sigma \Leftrightarrow \Delta \chi^{2}=9$.

The main difficulties we have to solve are the following: to find all possible solutions, to eliminate the mirage solutions, to find the most robust solutions.

One parameter allows us to determine the possible solutions. This fundamental parameter is the ratio $T_{\mathrm{p}} / T_{\mathrm{b}}$, where $T_{\mathrm{p}}$ and $T_{\mathrm{b}}$ are the precession period of the accretion disk and the binary period of the BBH system, respectively.

To find the possible solutions, for a given inclination angle and for several values of $T_{\mathrm{p}} / T_{\mathrm{b}}$ (say $T_{\mathrm{p}} / T_{\mathrm{b}}=10,30,100 \ldots$ ), we calculate the function $\chi^{2}\left(M_{1} / M_{2}\right)$, for $10^{-7} \leq M_{1} / M_{2} \leq 100$ in a first step, and then for a given value of $M_{1} / M_{2}$ determined previously, we calculate $\chi^{2}\left(T_{\mathrm{p}} / T_{\mathrm{b}}\right)$ for $1 \leq T_{\mathrm{p}} / T_{\mathrm{b}} \leq 10000$. The determination of $M_{1} / M_{2}$ does not depend on the choice of the inclination angle. Because we investigate a wide range for the parameters $M_{1} / M_{2}$ and $T_{\mathrm{p}} / T_{\mathrm{b}}$, we expect to be able to find all possible solutions.

In a second step, for different values of $M_{1} / M_{2}$ found previously, assuming that $T_{\mathrm{p}} / T_{\mathrm{b}}$ is a free parameter, we calculate $\chi^{2}\left(i_{0}\right)$. When the solution is found, it is not unique, but is a family of solutions. The solution shows a degeneracy, and we show below that the parameter for studying the degeneracy, that is, to find the range of parameters that provide the family of solutions, is $V_{\mathrm{a}}$, the propagation speed of the perturbation along the beam.

Generally, for any value of the parameters, the surface $\chi^{2}(\lambda)$, where $\lambda$ can be any of the free parameters of the system, $i_{\mathrm{o}}$, $M_{1} / M_{2}$, or $T_{\mathrm{p}} / T_{\mathrm{b}}$, for instance. is convex and does not present a minimum. Moreover, when we are in the convex part of the surface $\chi^{2}(\lambda)$, one of the important parameters of the problem can diverge. The important parameters of the problem that can diverge are: the bulk Lorentz factor of the $e^{ \pm}$beam, which has to be $\gamma_{\mathrm{c}} \leq 30$. This limit is imposed by the stability criterion for the propagation of the relativistic beam in the subrelativistic $\mathrm{e}^{-}-\mathrm{p}$ jet; the radius of the accretion disk, which has to be smaller than the distance between the distance between the $\mathrm{BH}$ and the first Lagrange point (see Sect. 3.1); the ratio $T_{\mathrm{p}} / T_{\mathrm{b}}$, which has to be $>1$, when $T_{\mathrm{p}} / T_{\mathrm{b}}=1$ the radius of the accretion disk becomes equal to the separation of the BBH system; and the total mass of the BBH system. 


\section{Appendix B: Fit of component C6}

We studied the three different models given in Table 2 . We calculated the curves $\chi^{2}\left(i_{\mathrm{o}}\right)$, which corresponds to the $\chi^{2}$ of the model of the ejection of the VLBI component for a given inclination angle $i_{\mathrm{o}}$.

\section{B.1. Precession model: Origin Co}

In this section we model the ejection of the VLBI component C6 assuming a single spinning $\mathrm{BH}$, that the VLBI ejection is perturbed by the precession of the accretion disk, and that the ejection origin is core $\mathrm{C} 0$. The direction for the precession rotation is chosen to be + .

We found that the possible range for the inclination angle is limited to $2^{\circ} \leq i_{\mathrm{o}} \leq 11.6^{\circ}$. The bulk Lorentz factor $\gamma_{\mathrm{c}}$ exceeds 30 and diverges when $i_{\mathrm{o}}>11.6^{\circ}$. The curve $\gamma_{\mathrm{c}}\left(i_{\mathrm{o}}\right)$ is shown in Fig. B.1.

\section{B.2. BBH CO-C9 model: Origin CO}

In this section we model the ejection of the VLBI component C6 assuming a $\mathrm{BBH}$ system that consists of core $\mathrm{C} 0$ and the stationary component $\mathrm{C} 9$. Its separation is $R_{\mathrm{C} 0-\mathrm{C} 9}=0.3$ mas, the VLBI ejection is perturbed by the precession of the accretion disk and the motion of the $\mathrm{BHs}$, and the ejection origin is core $\mathrm{C} 0$. The directions for the precession rotation and the $\mathrm{BBH}$ rotation are chosen to be + and - , respectively.

Following the method given in Appendix A.5, we calculated the function $\chi^{2}\left(M_{\mathrm{C} 0} / M_{\mathrm{C} 9}\right)$ for $i_{\mathrm{o}}=8^{\circ}$ and $T_{\mathrm{p}} / T_{\mathrm{b}}=10$. We found that the mass ratio is $0.1 \leq M_{\mathrm{C} 0} / M_{\mathrm{C} 9}<1$ (see Fig. B.3), that is, component $\mathrm{C} 6$ ejected from core $\mathrm{C} 0$ is ejected by the smallest $\mathrm{BH}$ of the $\mathrm{BBH}$ system $\mathrm{C} 0-\mathrm{C} 9$. It is possible to constrain and determine the mass ratio $M_{\mathrm{C} 0} / M_{\mathrm{C} 9}$ when the radius of the accretion disk around $\mathrm{C} 0, R_{\text {disk,C0 }}$ is smaller than the distance $D_{\mathrm{L} 1-\mathrm{C} 0}$ to obtain a stable solution (see Sect. 3.1 and Eqs. (2) and (3)).

Then we calculated $\chi_{2}\left(T_{\mathrm{p}} / T_{\mathrm{b}}\right)$ and $\chi^{2}\left(i_{\mathrm{o}}\right)$ for various values of $M_{\mathrm{C} 0} / M_{\mathrm{C} 9}$ and found that solutions with $M_{\mathrm{C} 0} / M_{\mathrm{C} 9} \geq 0.6$ have $R_{\text {disk,C0 }} \geq D_{\mathrm{L} 1-\mathrm{C} 0}$ and do not correspond to stable solutions. Moreover, we found that for solutions with $M_{\mathrm{C} 0} / M_{\mathrm{C} 9} \leq 0.5$ the solution with $M_{\mathrm{C} 0} / M_{\mathrm{C} 9} \approx 0.5$ has the smallest $\chi_{\min }^{2}$, that is, the solution that provides the best fit of the VLBI component $\mathrm{C} 6$ corresponds to the $\mathrm{BBH}$ system $\mathrm{C} 0-\mathrm{C} 9$ characterized by $R_{\mathrm{C} 0-\mathrm{C} 9}=0.3$ mas and $M_{\mathrm{C} 0} / M_{\mathrm{C} 9}=0.5$. The corresponding solution is characterized by $i_{\mathrm{o}} \approx 6.7^{\circ}, T_{\mathrm{p}} / T_{\mathrm{b}} \approx 5.5$ and $\Omega \approx 3.1^{\circ}$.

The curves $\chi^{2}\left(i_{0}\right)$ corresponding to the mass ratios $M_{\mathrm{C} 0} / M_{\mathrm{C} 9}=0.1,0.5$ and 0.8 are shown in Fig. B.4. The curves $\left(R_{\text {disk,C0 }} / D_{\mathrm{L} 1-\mathrm{C} 0}\right)\left(i_{\mathrm{o}}\right)$ corresponding to the mass ratios $M_{\mathrm{C} 0} / M_{\mathrm{C} 9}=0.1,0.5$ and 0.8 are shown in Fig. B.5.

We found that the curve $\chi^{2}\left(i_{0}\right)$ is convex. It does not have a minimum, that is, there is no stable solution. The curve $\chi^{2}\left(i_{0}\right)$ is shown in Fig. B.2.

\section{B.2.1. Separation of the BBH system $\mathrm{C} 0-\mathrm{C} 9$}

We showed in Sect. 2.4 that from the mean position of $\mathrm{C} 9$, that is, $X_{\mathrm{C} 9}=-0.082 \pm 0.025$ mas and $Y_{\mathrm{C} 9}=-0.284 \pm 0.061$ mas, the distance between the core $\mathrm{C} 0$ and $\mathrm{C} 9$ should be $R_{\mathrm{C} 0-\mathrm{C} 9}=$ $0.296 \pm 0.066$ mas. However, the solution corresponding to $R_{\mathrm{C} 0-\mathrm{C} 9}=0.300$ mas is characterized by $R_{\mathrm{disk}, \mathrm{C} 0} / D_{\mathrm{L} 1-\mathrm{C} 0}=0.85$, and when we reduce $R_{\mathrm{C} 0-\mathrm{C} 9}$, we will have $R_{\text {disk, } \mathrm{C} 0} / D_{\mathrm{L} 1-\mathrm{C} 0}>1$. We found that we must have $R_{\mathrm{C} 0-\mathrm{C} 9} \geq 0.280$ mas, thus the distance between C0 and C9 is $R_{\mathrm{C} 0-\mathrm{C} 9}=0.296_{-0.020}^{+0.066}$ mas, that is, the errors are asymmetrical.

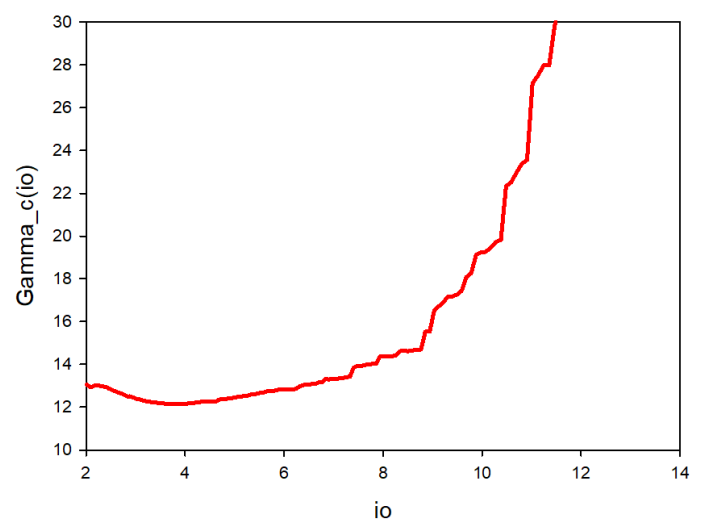

Fig. B.1. Precession model. Component $\mathrm{C} 6$ is ejected from core $\mathrm{C} 0$ : the bulk Lorentz factor $\gamma_{\mathrm{c}}\left(i_{\mathrm{o}}\right)$.

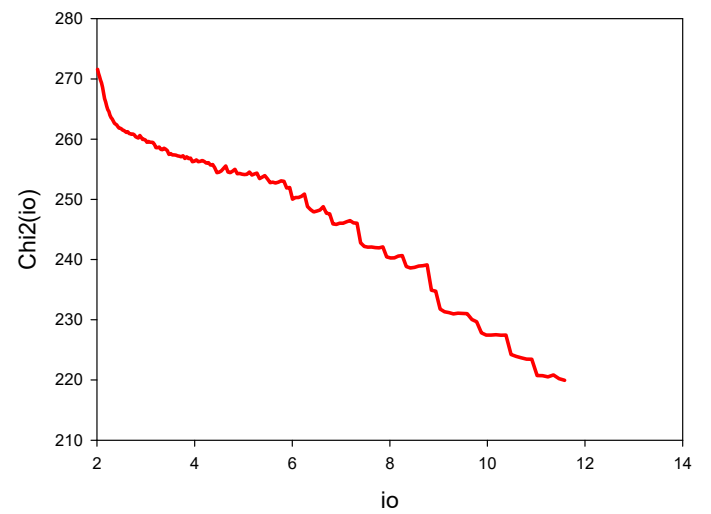

Fig. B.2. Precession model. Component C6 is ejected from core C0: the function $\chi^{2}\left(i_{0}\right)$. The curve $\chi^{2}\left(i_{0}\right)$ is convex.

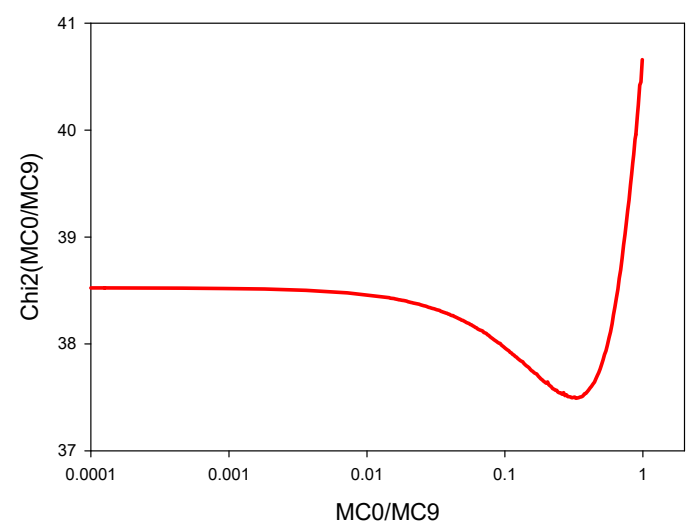

Fig. B.3. BBH model $\mathrm{C} 0-\mathrm{C} 9$ characterized by $R_{\mathrm{C} 0-\mathrm{C} 9}=0.3$ mas. Component $\mathrm{C} 6$ is ejected from core $\mathrm{C} 0$ : the function $\chi^{2}\left(M_{\mathrm{C} 0} / M_{\mathrm{C} 9}\right)$. The curve $\chi^{2}\left(M_{\mathrm{C} 0} / M_{\mathrm{C} 9}\right)$ obtained for $i_{\mathrm{o}}=8^{\circ}$ and $T_{\mathrm{p}} / T_{\mathrm{b}}=10$. The mass ratio is $0.1 \leq M_{\mathrm{C} 0} / M_{\mathrm{C} 9}<1$.

When the solution is known, we can determine the best distance between $\mathrm{C} 0$ and $\mathrm{C} 9$ and its error. We can choose 30 points, for example, whose positions are close to the $\mathrm{C} 9$ position within the errors $\Delta X=0.025$ mas and $\Delta Y=0.061$ mas. For each point, we calculate its new distance to $\mathrm{C} 0$ and the new offset of the VLBI data, and then we calculate the function $\chi^{2}\left(i_{\mathrm{o}}\right)$. Then we will be able to deduce the best value of the distance between the two BHs and its error. However, this is beyond the scope of the article. 


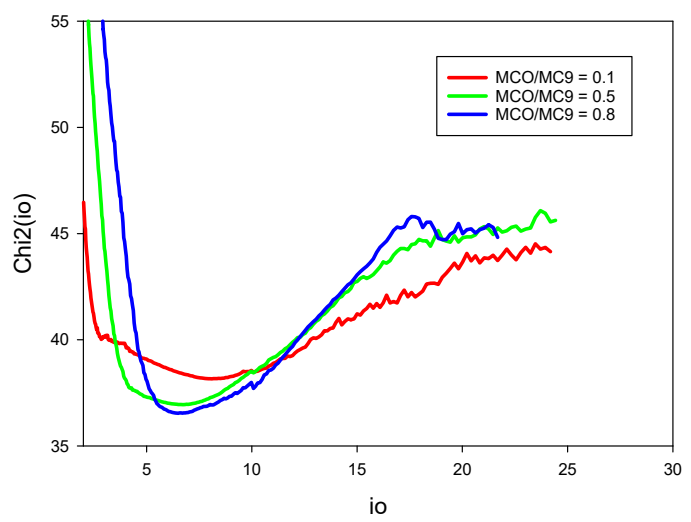

Fig. B.4. $\mathrm{BBH}$ model $\mathrm{C} 0-\mathrm{C} 9$ characterized by $R_{\mathrm{C} 0-\mathrm{C} 9}=0.3$ mas. Component $\mathrm{C} 6$ is ejected from core $\mathrm{C} 0$ : the functions $\chi^{2}\left(i_{0}\right)$. The curves $\chi^{2}\left(i_{0}\right)$ obtained for different values of the mass ratios $M_{\mathrm{C} 0} / M_{\mathrm{C} 9}=0.1$, 0.5 and 0.8 . All the curves are concave and show a minimum, but the solution corresponding to $M_{\mathrm{C} 0} / M_{\mathrm{C} 9}=0.8$ is not stable because the corresponding ratio $\left(R_{\text {disk,C0 }} / D_{\mathrm{L} 1-\mathrm{C} 0}\right)\left(i_{\mathrm{o}}\right)$ is higher than 1 (see Fig. B.5). For solutions with $M_{\mathrm{C} 0} / M_{\mathrm{C} 9} \leq 0.5$ the solution with $M_{\mathrm{C} 0} / M_{\mathrm{C} 9} \approx 0.5$ has the smallest $\chi_{\min }^{2}$, i.e., the solution that provides the best fit of the VLBI component $\mathrm{C} 6$ corresponds to the $\mathrm{BBH}$ system $\mathrm{C} 0-\mathrm{C} 9$ characterized by $R_{\mathrm{C} 0-\mathrm{C} 9} \approx 0.3$ mas and $M_{\mathrm{C} 0} / M_{\mathrm{C} 9} \approx 0.5$.

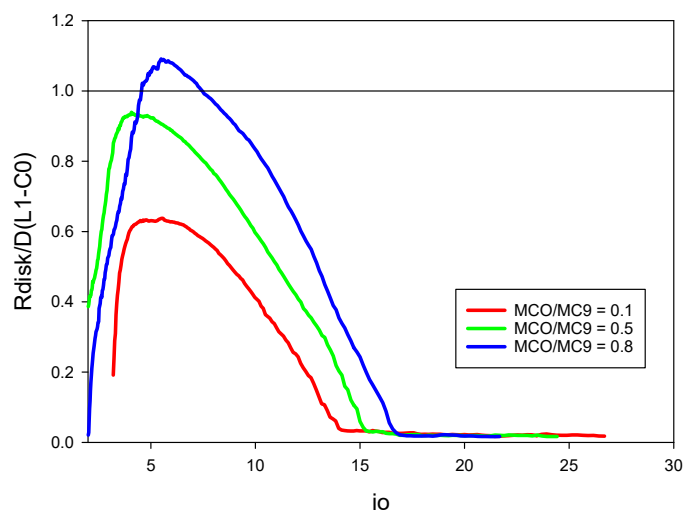

Fig. B.5. $\mathrm{BBH}$ model $\mathrm{C} 0-\mathrm{C} 9$ characterized by $R_{\mathrm{C} 0-\mathrm{C} 9}=0.3$ mas. Component $\mathrm{C} 6$ is ejected from core $\mathrm{C} 0$ : the functions $\left(R_{\text {disk,C0}} / D_{\mathrm{L} 1-\mathrm{C} 0}\right)\left(i_{\mathrm{o}}\right)$. The curves $\left(R_{\mathrm{disk}, \mathrm{C} 0} / D_{\mathrm{L} 1-\mathrm{C} 0}\right)\left(i_{\mathrm{o}}\right)$ corresponding to the mass ratios $M_{\mathrm{C} 0} / M_{\mathrm{C} 9}=0.1,0.5$ and 0.8 are shown. Solutions with $M_{\mathrm{C} 0} / M_{\mathrm{C} 9} \geq 0.6$ have $R_{\text {disk,C0 }} \geq D_{\mathrm{L} 1-\mathrm{C} 0}$ and do not correspond to stable solutions.

\section{B.2.2. Solution family}

Using the parameters of the solution, we gradually varied $V_{\mathrm{a}}$ between $0.001 c$ and $0.45 c$. The function $\chi^{2}\left(V_{\mathrm{a}}\right)$ remained constant, indicating a degeneracy of the solution (see Fig. B.6). The corresponding ranges of the parameters of the $\mathrm{BBH}$ system are given in Sect. 3.4.

\section{B.3. $\mathrm{BBH}$ model $\mathrm{CO}-\mathrm{C} 3$ : Origin $\mathrm{CO}$}

In this section we model the ejection of the VLBI component C6 assuming the $\mathrm{BBH}$ system consists of core $\mathrm{C} 0$ and the stationary component C3. Its separation is $R_{\mathrm{C} 0-\mathrm{C} 3}=0.56$ mas, the VLBI ejection is perturbed by the precession of the accretion disk and the motion of the $\mathrm{BHs}$, and the ejection origin is $\mathrm{C} 0$. The directions for the precession rotation and the $\mathrm{BBH}$ rotation are chosen to be + and - , respectively.

We searched for the solution with $R_{\mathrm{C} 0-\mathrm{C} 3}=0.56$ mas and $M_{\mathrm{C} 0} / M_{\mathrm{C} 3}=0.5$ to compare it with the solution found previously in Appendix B.2. The curve $\chi^{2}\left(i_{\mathrm{o}}\right)$ is shown in Fig. B.7. It

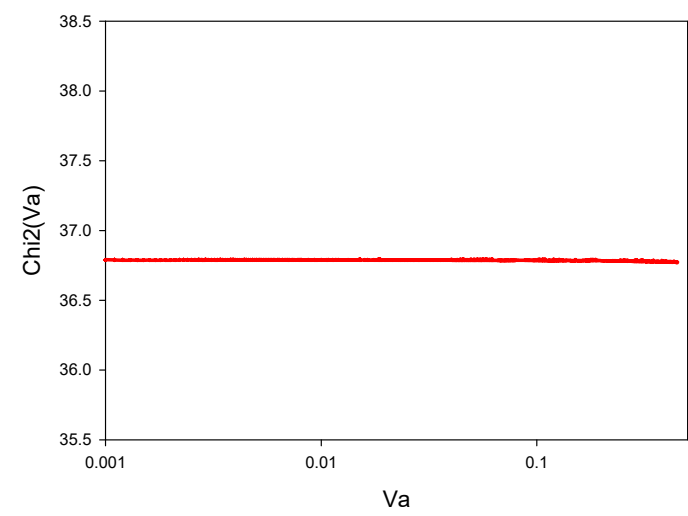

Fig. B.6. $\mathrm{BBH}$ model $\mathrm{C} 0-\mathrm{C} 9$ characterized by $R_{\mathrm{C} 0-\mathrm{C} 9}=0.3$ mas and $M_{\mathrm{C} 0} / M_{\mathrm{C} 9}=0.5$. Component $\mathrm{C} 6$ is ejected from the core $\mathrm{C} 0$ : the function $\chi^{2}\left(V_{\mathrm{a}}\right) \cdot \chi^{2}\left(V_{\mathrm{a}}\right)$ remained constant, indicating a degeneracy of the solution.

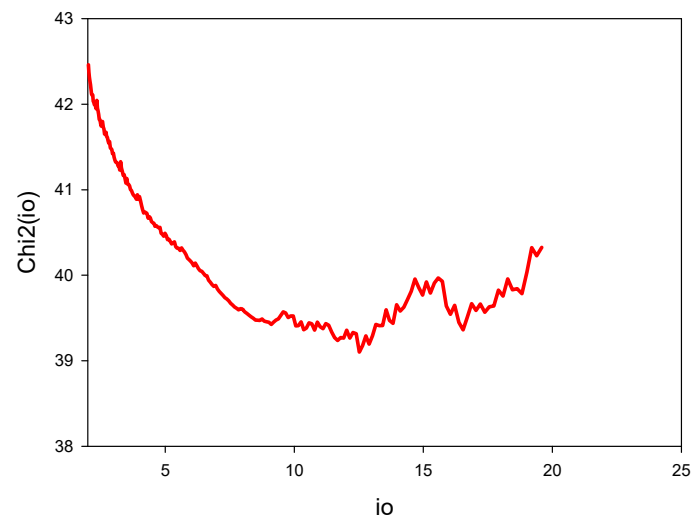

Fig. B.7. $\mathrm{BBH}$ model $\mathrm{C} 0-\mathrm{C} 3$ characterized by $R_{\mathrm{C} 0-\mathrm{C} 3} \approx 0.56$ mas and $M_{\mathrm{C} 0} / M_{\mathrm{C} 3} \approx 0.5$. Component C6 is ejected from core $\mathrm{C} 0$ : the function $\chi^{2}\left(i_{\mathrm{o}}\right)$. The curve $\chi^{2}\left(i_{\mathrm{o}}\right)$ has a minimum, but the corresponding solution has a $\chi_{\min }^{2}$ larger than the $\chi_{\min }^{2}$ of the solution of the fit of C6 assuming it is ejected by the $\mathrm{BBH}$ system $\mathrm{C} 0-\mathrm{C} 9$ characterized by $R_{\mathrm{C} 0-\mathrm{C} 9} \approx 0.3$ mas and $M_{\mathrm{C} 0} / M_{\mathrm{C} 9}=0.5$ (see Fig. B.4). Moreover, it is a very weak solution, i.e., its robustness is $\leq 1$ (see Appendix A.5) and is rejected.

has a minimum, but the corresponding solution has a $\chi_{\min }^{2} \approx 39.1$ larger than the $\chi_{\min }^{2} \approx 36.9$ of the solution of the fit of C6 assuming it is ejected by the $\mathrm{BBH}$ system $\mathrm{C} 0-\mathrm{C} 9$ characterized by $R_{\mathrm{C} 0-\mathrm{C} 9}=0.3$ mas and $M_{\mathrm{C} 0} / M_{\mathrm{C} 9}=0.5$ (see Fig. B.4). Moreover, it is a very weak solution, that is, its robustness is $\leq 1$ (see Appendix A.5) and is rejected.

\section{Appendix C: Fit of component $\mathbf{C 1 3}$}

We studied the four different cases given in Table 4. We calculated the curves $\chi^{2}\left(i_{0}\right)$.

\section{C.1. Precession model: Origin CO}

In this section we model the ejection of the VLBI component $\mathrm{C} 13$ assuming a single spinning $\mathrm{BH}$, the VLBI ejection is perturbed by the precession of the accretion disk, and the ejection origine is core $\mathrm{C} 0$. The direction for the precession rotation is chosen to be + .

We found that the possible range for the inclination angle is limited to $2^{\circ} \leq i_{\mathrm{o}} \leq 13.9^{\circ}$. The bulk Lorentz factor $\gamma_{\mathrm{c}}$ exceeds 30 and diverges if $i_{\mathrm{o}}>13.9^{\circ}$. The curve $\gamma_{\mathrm{c}}\left(i_{\mathrm{o}}\right)$ is shown in Fig. C.1. 


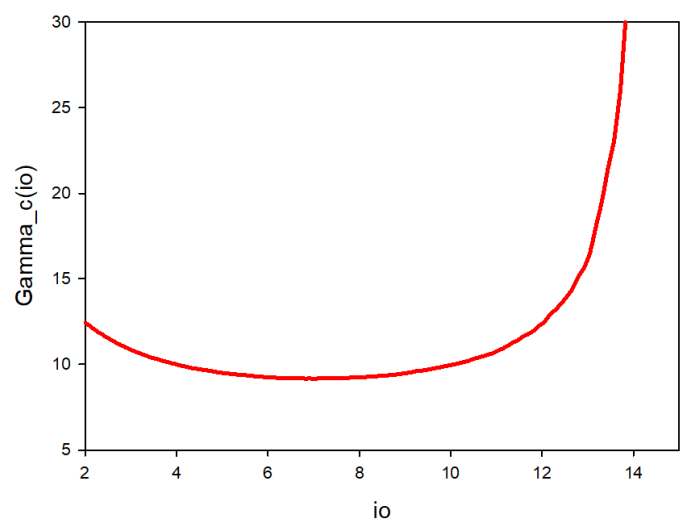

Fig. C.1. Precession model. Component $\mathrm{C} 13$ is ejected from the core C0: the bulk Lorentz factor $\gamma_{\mathrm{c}}\left(i_{\mathrm{o}}\right)$.

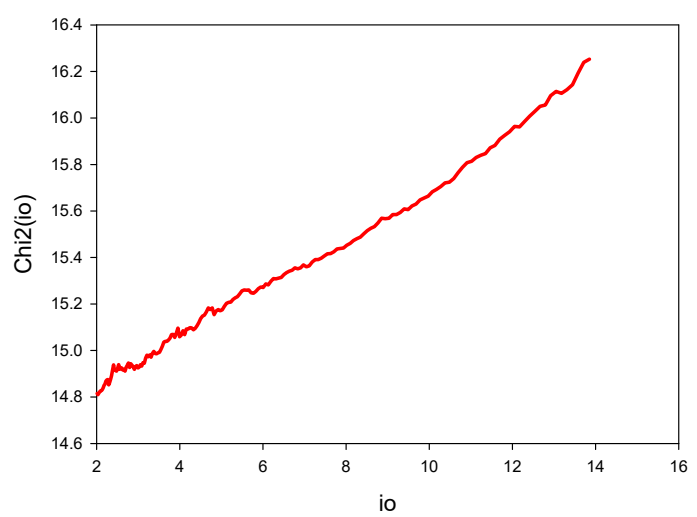

Fig. C.2. Precession model. Component $\mathrm{C} 13$ is ejected from the core $\mathrm{C} 0$ : the function $\chi^{2}\left(i_{0}\right)$. The curve $\chi^{2}\left(i_{0}\right)$ is convex, it does not have a minimum, i.e., there is no stable solution.

We found that the curve $\chi^{2}\left(i_{0}\right)$ is convex, it does not have a minimum, that is, there is no stable solution. The curve $\chi^{2}\left(i_{0}\right)$ is shown in Fig. C.2.

\section{C.2. Precession model: origin C9}

In this section we mode the ejection of the VLBI component $\mathrm{C} 13$ assuming a single spinning $\mathrm{BH}$, the VLBI ejection is perturbed by the precession of the accretion disk, and the ejection origin is the stationary component $\mathrm{C} 9$. The direction for the precession rotation is chosen to be + .

We found that the possible range for the inclination angle is limited to $2^{\circ} \leq i_{\mathrm{o}} \leq 14.7^{\circ}$. The bulk Lorentz factor $\gamma_{\mathrm{c}}$ exceeds 30 and diverges when $i_{\mathrm{o}}>14.7^{\circ}$. The curve $\gamma_{\mathrm{c}}\left(i_{\mathrm{o}}\right)$ is shown in Fig. C.3.

We found that the curve $\chi^{2}\left(i_{0}\right)$ is convex, it does not have a minimum, that is, there is no stable solution. The curve $\chi^{2}\left(i_{0}\right)$ is shown in Fig. C.4.

\section{C.3. BBH model C0-C9: Origin C9}

In this section we model the ejection of the VLBI component $\mathrm{C} 13$ assuming that the ejection origin is the stationary component $\mathrm{C} 9$ and that it is ejected by the same $\mathrm{BBH}$ system that has ejected $\mathrm{C} 6$, that is, the $\mathrm{BBH}$ system $\mathrm{C} 0-\mathrm{C}$, whose separation is $R_{\mathrm{C} 0-\mathrm{C} 9}=0.3$ mas. The VLBI ejection is perturbed by the precession of the accretion disk and the motion of the BHs, and the ejection origin is core $\mathrm{C} 0$. The directions for the preces-

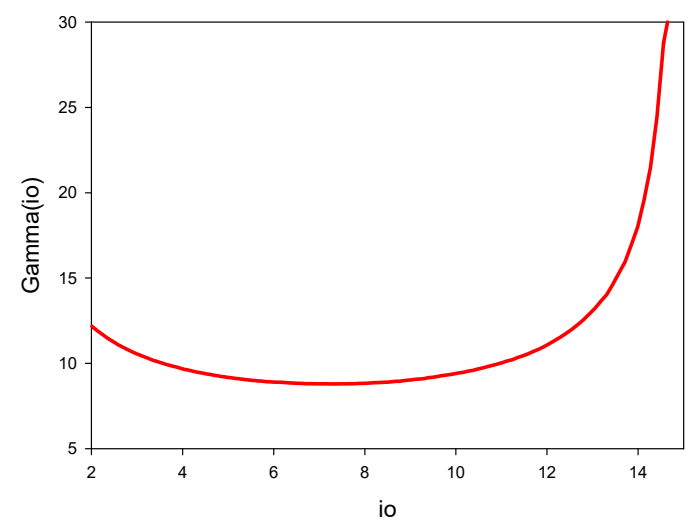

Fig. C.3. Precession model. Component $\mathrm{C} 13$ is ejected from the stationary component C9: the bulk Lorentz factor $\gamma_{\mathrm{c}}\left(i_{\mathrm{o}}\right)$.

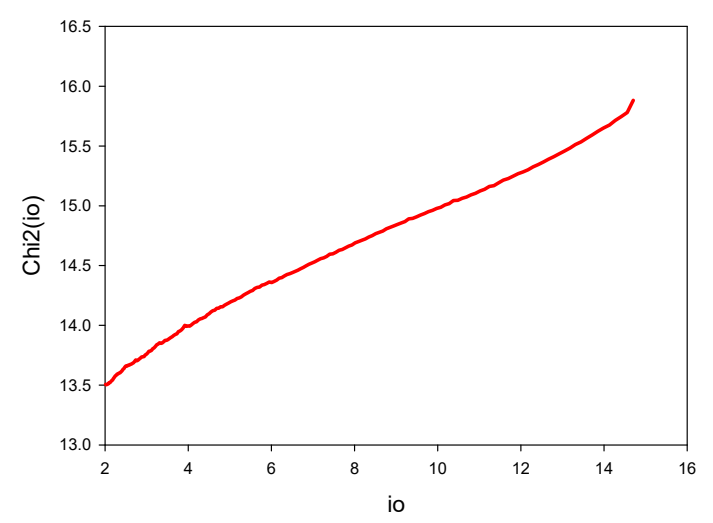

Fig. C.4. Precession model. Component $\mathrm{C} 13$ is ejected from the stationary component C9: the function $\chi^{2}\left(i_{\mathrm{o}}\right)$. The curve $\chi^{2}\left(i_{\mathrm{o}}\right)$ is convex, it does not have a minimum, i.e., there is no stable solution.

sion rotation and the $\mathrm{BBH}$ rotation are chosen to be + and - , respectively.

First, we calculated the function $\chi^{2}\left(M_{\mathrm{C} 0} / M_{\mathrm{C} 9}\right)$ for $i_{\mathrm{O}}=8^{\circ}$ and $T_{\mathrm{p}} / T_{\mathrm{b}}=10$. We found that the mass ratio is $M_{\mathrm{C} 9} / M_{\mathrm{C} 0} \geq 1.0$ (see Fig. C.5), that is, component $\mathrm{C} 13$ ejected from the stationary component $\mathrm{C} 9$ is ejected by the largest $\mathrm{BH}$ of the $\mathrm{BBH}$ system. It is possible to constrain and determine the mass ratio $M_{\mathrm{C} 9} / M_{\mathrm{C} 0}$ when the radius of the accretion disk around C9, $R_{\text {disk,C9 }}$ is smaller than the distance $D_{\mathrm{L} 1-\mathrm{C} 9}$ to obtain a stable solution (see Sect. 3.1).

Then we calculated $\chi_{2}\left(T_{\mathrm{p}} / T_{\mathrm{b}}\right)$ and $\chi^{2}\left(i_{\mathrm{o}}\right)$ for various values of the mass ratio $M_{\mathrm{C} 9} / M_{\mathrm{C} 0}$, that is, $M_{\mathrm{C} 9} / M_{\mathrm{C} 0}=1$, 2 , and 3 . The curves corresponding to $\chi^{2}\left(i_{\mathrm{o}}\right)$ are shown in Fig. C.6. We found that solutions with $M_{\mathrm{C} 9} / M_{\mathrm{C} 0} \geq 3.0$ have $R_{\text {disk,C } 9} \geq D_{\mathrm{L} 1-\mathrm{C} 0}$ and do not correspond to stable solutions. The curves $\left(R_{\text {disk,C } 9} / D_{\mathrm{L} 1-\mathrm{C} 9}\right)\left(i_{\mathrm{o}}\right)$ corresponding to the mass ratios $M_{\mathrm{C} 9} / M_{\mathrm{C} 0}=1,2$, and 3 are shown in Fig. C.7. Moreover, we found that for solutions with $M_{\mathrm{C} 9} / M_{\mathrm{C} 0} \leq 2.0$ the solution with $M_{\mathrm{C} 9} / M_{\mathrm{C} 0}=2.0$ has the smallest $\chi_{\min }^{2}$, that is, the solution that provides the best fit of the VLBI component $\mathrm{C} 13$ corresponds to the $\mathrm{BBH}$ system $\mathrm{C} 0-\mathrm{C} 9$ with $R_{\mathrm{C} 0-\mathrm{C} 9}=0.3$ mas and $M_{\mathrm{C} 9} / M_{\mathrm{C} 0}=2.0 \pm 0.5$ assuming that component $\mathrm{C} 13$ is ejected from the stationary component $\mathrm{C} 9$. The solution is characterized by by the inclination angle $i_{\mathrm{o}} \approx 5.8^{\circ}$, the ratio $T_{\mathrm{p}} / T_{\mathrm{b}} \approx 10.2$, and the angle $\Omega \approx 3.3^{\circ}$.

Using the parameters of the solution, we gradually varied $V_{\mathrm{a}}$ between $0.001 \mathrm{c}$ and $0.45 \mathrm{c}$. The function $\chi^{2}\left(V_{\mathrm{a}}\right)$ remained constant, indicating a degeneracy of the solution (see Fig. C.8). 


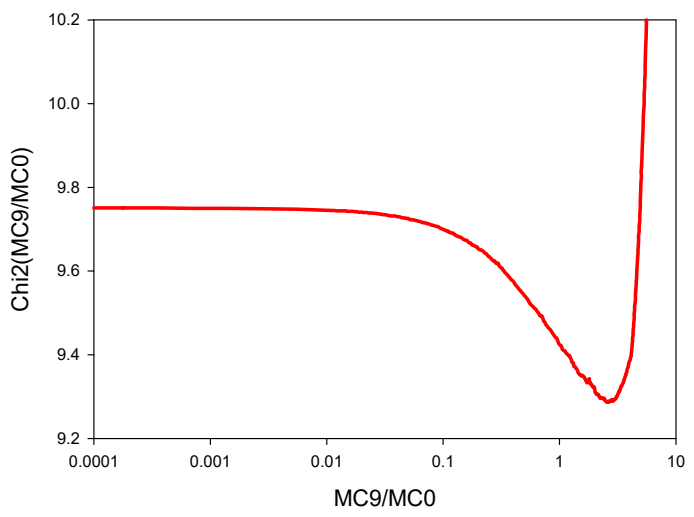

Fig. C.5. BBH model $\mathrm{C} 0-\mathrm{C} 9$ characterized by $R_{\mathrm{C} 0-\mathrm{C} 9} \approx 0.3$ mas. Component $\mathrm{C} 13$ is ejected from the stationary component $\mathrm{C} 9$ : the function $\chi^{2}\left(M_{\mathrm{C} 9} / M_{\mathrm{C} 0}\right)$ obtained for $i_{\mathrm{o}}=8^{\circ}$ and $T_{\mathrm{p}} / T_{\mathrm{b}}=10$. The mass ratio is $M_{\mathrm{C} 9} / M_{\mathrm{C} 0} \geq 1.0$.

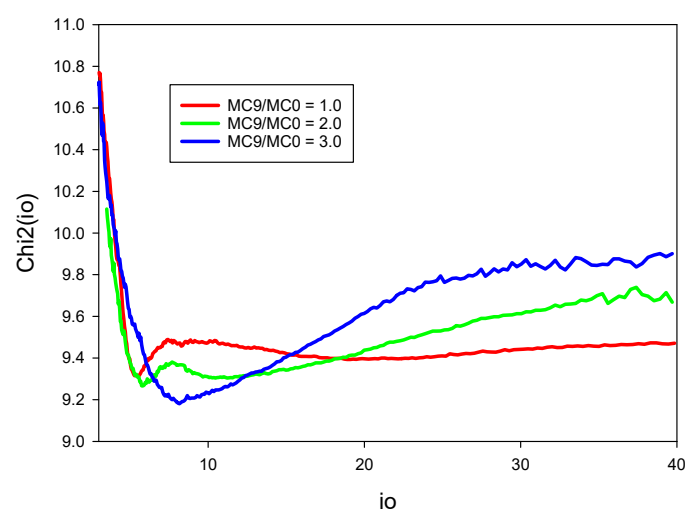

Fig. C.6. $\mathrm{BBH}$ model $\mathrm{C} 0-\mathrm{C} 9$ characterized by $R_{\mathrm{C} 0-\mathrm{C} 9}=0.3$ mas. Component $\mathrm{C} 13$ is ejected from the stationary component $\mathrm{C} 9$ : the functions $\chi^{2}\left(i_{0}\right)$ obtained for different values of the mass ratios $M_{\mathrm{C} 9} / M_{\mathrm{C} 0}=1,2$ and 3. All the curves are concave and show a minimum, but solutions corresponding to $M_{\mathrm{C} 9} / M_{\mathrm{C} 0} \geq 3$ are not stable because the corresponding mass ratios $\left(R_{\text {disk,C9}} / D_{\mathrm{L} 1-\mathrm{C} 9}\right)\left(i_{\mathrm{o}}\right)$ are higher than 1 (see Fig. C.7).

The corresponding ranges of the parameters of the BBH system are given in Sect. 3.5.

\section{C.4. BBH model C0-C9: Origin CO}

In this section we model the ejection of the VLBI component $\mathrm{C} 13$ assuming that the ejection origin is core $\mathrm{C} 0$ and that it is ejected by the same BBH system that has ejected $\mathrm{C} 6$, that is, the $\mathrm{BBH}$ system $\mathrm{C} 0-\mathrm{C} 9$, whose separation is $R_{\mathrm{C} 0-\mathrm{C} 9}=0.3$ mas, and the mass ratio is $M_{\mathrm{C} 0} / M_{\mathrm{C} 9}=0.5$. The VLBI ejection is perturbed by the precession of the accretion disk and the motion of the $\mathrm{BHs}$, and the ejection origin is core $\mathrm{C} 0$. The directions for the precession rotation and the $\mathrm{BBH}$ rotation are chosen to be + and - , respectively.

First, we calculated the function $\chi^{2}\left(M_{\mathrm{C} 0} / M_{\mathrm{C} 9}\right)$ for $i_{0}=8^{\circ}$ and $T_{\mathrm{p}} / T_{\mathrm{b}}=100$. We found that the mass ratio is $1.0 \leq$ $M_{\mathrm{C} 0} / M_{\mathrm{C} 9} \leq 10$ (see Fig. C.9), that is, component $\mathrm{C} 13$ ejected from the core is ejected by the largest $\mathrm{BH}$ of the $\mathrm{BBH}$ system. This result contradicts the result found for the mass ratio $M_{\mathrm{C} 0} / M_{\mathrm{C} 9} \approx 0.5$. during the fit of component $\mathrm{C} 6$ ejected from the core $\mathrm{C} 0$ of the same BBH system C0-C9 (see Appendix B.2). This result shows already that component $\mathrm{C} 13$ cannot be ejected from core $\mathrm{C} 0$ of the $\mathrm{BBH}$ system $\mathrm{C} 0-\mathrm{C} 9$.

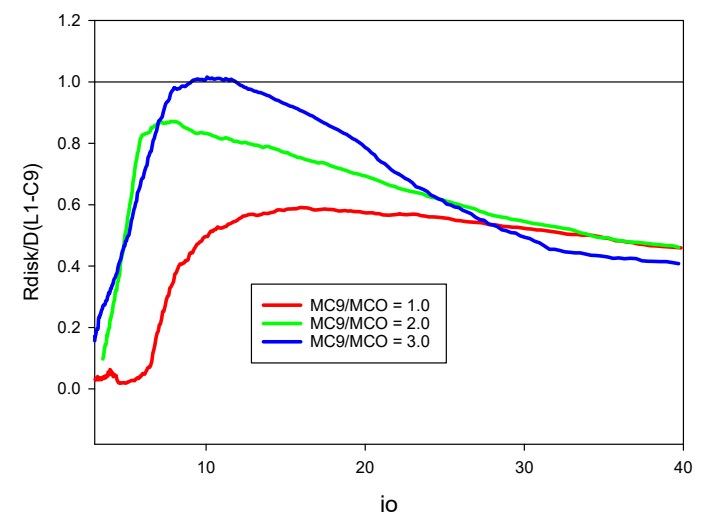

Fig. C.7. BBH model $\mathrm{C} 0-\mathrm{C} 9$ characterized by $R_{\mathrm{C} 0-\mathrm{C} 9}=0.3$ mas. Component $\mathrm{C} 13$ is ejected from the stationary component $\mathrm{C} 9$ : the functions $\left(R_{\text {disk,C9}} / D_{\mathrm{L} 1-\mathrm{C} 09}\right)\left(i_{\mathrm{o}}\right)$ corresponding to the mass ratios $M_{\mathrm{C} 9} / M_{\mathrm{C} 0}=1,2$, and 3. Solutions with $M_{\mathrm{C} 9} / M_{\mathrm{C} 0} \geq 3$ have $R_{\mathrm{disk}, \mathrm{C} 9} \geq D_{\mathrm{L} 1-\mathrm{C} 9}$ and do not correspond to stable solutions.

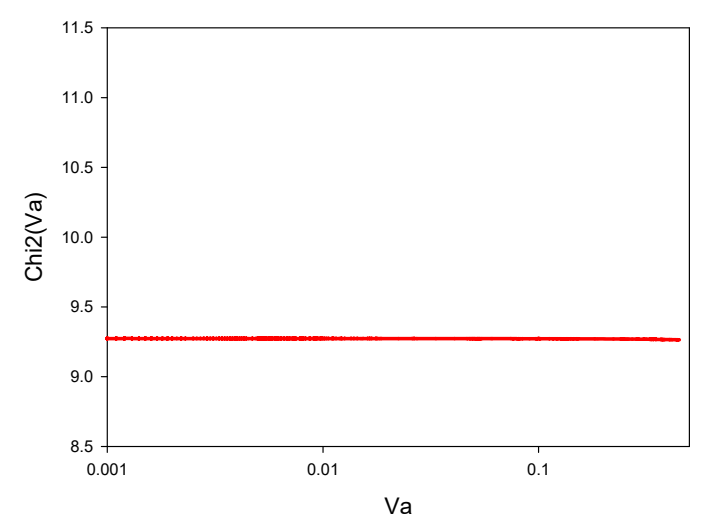

Fig. C.8. BBH model C0-C9 characterized by $R_{\mathrm{C} 0-\mathrm{C} 9}=0.3$ mas and $M_{\mathrm{C} 9} / M_{\mathrm{C} 0}=2$. Component $\mathrm{C} 13$ is ejected from the stationary component C9: the function $\chi^{2}\left(V_{\mathrm{a}}\right) \cdot \chi^{2}\left(V_{\mathrm{a}}\right)$ remained constant, indicating a degeneracy of the solution.

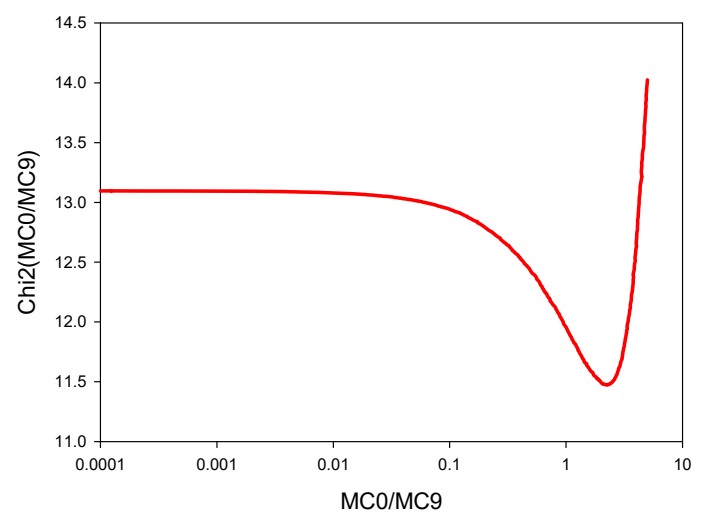

Fig. C.9. $\mathrm{BBH}$ model $\mathrm{C} 0-\mathrm{C} 9$ characterized by $R_{\mathrm{C} 0-\mathrm{C} 9} \approx 0.3$ mas. Component $\mathrm{C} 13$ is ejected from core $\mathrm{C} 0$ : the function $\chi^{2}\left(M_{\mathrm{C} 0} / M_{\mathrm{C} 9}\right)$ obtained for $i_{\mathrm{o}}=8^{\circ}$ and $T_{\mathrm{p}} / T_{\mathrm{b}}=100$. The mass ratio is $1.0 \leq M_{\mathrm{C} 0} / M_{\mathrm{C} 9}<10.0$. This result shows that component $\mathrm{C} 13$ cannot be ejected from core $\mathrm{C} 0$ of the $\mathrm{BBH}$ system $\mathrm{C} 0-\mathrm{C} 9$.

However, we calculated $\chi_{2}\left(T_{\mathrm{p}} / T_{\mathrm{b}}\right)$ and $\chi^{2}\left(i_{\mathrm{o}}\right)$ for $M_{\mathrm{C} 0} / M_{\mathrm{C} 9}=0.5$ (the value of the mass ratio found during the fit of component C6). The curve $\chi^{2}\left(i_{0}\right)$ corresponding to the mass ratio $M_{\mathrm{C} 0} / M_{\mathrm{C} 9}=0.5$ is shown in Fig. C.10. The curve is convex and does not have a minimum, that is, there 


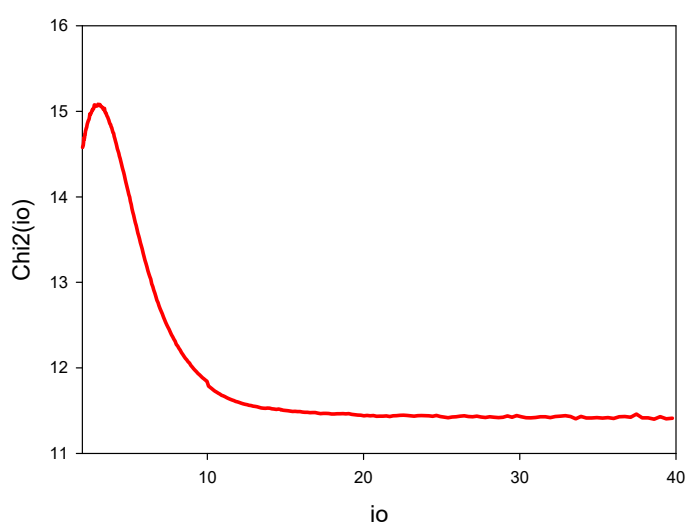

Fig. C.10. $\mathrm{BBH}$ model $\mathrm{C} 0-\mathrm{C} 9$ characterized by $R_{\mathrm{C} 0-\mathrm{C} 9}=0.3$ mas and the mass ratio $M_{\mathrm{C} 0} / M_{\mathrm{C} 9}=0.5$. Component $\mathrm{C} 13$ is ejected from the core $\mathrm{C} 0$ : the function $\chi^{2}\left(i_{0}\right)$. The curve $\chi^{2}\left(i_{0}\right)$ is convex and does not have a minimum, i.e., there is no solution. Thus component $\mathrm{C} 13$ cannot be ejected from core $\mathrm{C} 0$ of the $\mathrm{BBH}$ system $\mathrm{C} 0-\mathrm{C} 9$ characterized by the mass ratio $M_{\mathrm{C} 0} / M_{\mathrm{C} 9}=0.5$.

is no solution, and thus component $\mathrm{C} 13$ cannot be ejected from the core $\mathrm{C} 0$ of the $\mathrm{BBH}$ system $\mathrm{C} 0-\mathrm{C} 9$ characterized by $R_{\mathrm{C} 0-\mathrm{C} 9}=0.3$ mas and the mass ratio $M_{\mathrm{C} 0} / M_{\mathrm{C} 9}=0.5$.

\section{Appendix D: Fit of component C7}

We studied the three different models given in Table 6. We calculated the curves $\chi^{2}\left(i_{\mathrm{o}}\right)$.

\section{D.1. Precession model: Origin CO}

In this section we model the ejection of the VLBI component C7 assuming a single spinning $\mathrm{BH}$, that the VLBI ejection is perturbed by the precession of the accretion disk, and that the ejection origin is core $\mathrm{C} 0$. The direction for the precession rotation is chosen to be + .

We found that the possible range for the inclination angle is limited to $2^{\circ} \leq i_{\mathrm{o}} \leq 14.4^{\circ}$. The bulk Lorentz factor $\gamma_{\mathrm{c}}$ exceeds 30 and diverges when $i_{\mathrm{o}}>14.4^{\circ}$.

We found that the curve $\chi^{2}\left(i_{0}\right)$ is convex, it does not have a minimum, that is, there is no stable solution. The curve $\chi^{2}\left(i_{0}\right)$ is shown in Fig. D.2.

\section{D.2. $\mathrm{BBH}$ model $(\mathrm{CO}+\mathrm{C9})-\mathrm{C} 3$ : Origin $\mathrm{C} 3$}

In this section we model the ejection of the VLBI component $\mathrm{C} 7$ assuming that it is ejected by the $\mathrm{BBH}$ system formed by $\mathrm{C} 0+\mathrm{C} 9$ and $\mathrm{C} 3$, whose separation is $R_{\mathrm{G}-\mathrm{C} 3}=0.370$ mas (see Fig. 1). The VLBI ejection is perturbed by the precession of the accretion disk and the motion of the $\mathrm{BHs}$, and the ejection origin is component $\mathrm{C} 3$. The directions for the precession rotation and the $\mathrm{BBH}$ rotation are chosen to be + and - , respectively.

First, we calculated the function $\chi^{2}\left(M_{\mathrm{C} 0} / M_{\mathrm{C} 9}\right)$ for $i_{\mathrm{o}}=8^{\circ}$ and $T_{\mathrm{p}} / T_{\mathrm{b}}=10$. The solutions corresponding to the ejection of C7 are characterized by $M_{\mathrm{C} 3} /\left(M_{\mathrm{C} 0}+M_{\mathrm{C} 9}\right) \leq 0.1$, that is, the mass of the $\mathrm{BH}$ associated with component $\mathrm{C} 3$ is far lower than the mass $M_{\mathrm{C} 0}+M_{\mathrm{C} 9}$ (see Fig. D.1).

Then we calculated the curves $\chi^{2}\left(i_{\mathrm{o}}\right)$ corresponding to the separation $R_{\mathrm{G}-\mathrm{C} 3}=0.37$ mas and the mass ratios $M_{\mathrm{C} 3} /\left(M_{\mathrm{C} 0}+\right.$ $\left.M_{\mathrm{C} 9}\right)=0.005,0.01,0.05$, and 0.1 . We found that only solutions with $M_{\mathrm{C} 3} /\left(M_{\mathrm{C} 0}+M_{\mathrm{C} 9}\right)<0.05$ are possible and have a minimum (see Fig. D.2). Solutions with $M_{\mathrm{C} 3} /\left(M_{\mathrm{C} 0}+M_{\mathrm{C} 9}\right) \geq 0.05$ do not

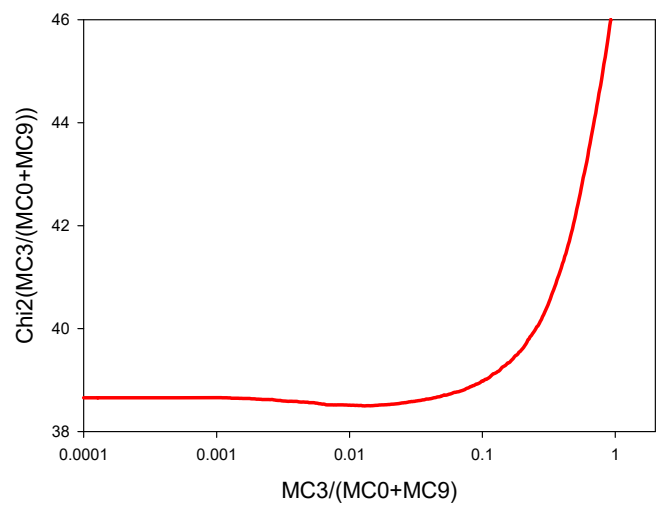

Fig. D.1. BBH model $(\mathrm{C} 0+\mathrm{C} 9)-\mathrm{C} 3$ characterized by $R_{\mathrm{G}-\mathrm{C} 3}=0.37$ mas. Component $\mathrm{C} 7$ is ejected from the stationary component $\mathrm{C} 3$ : the function $\chi^{2}\left(M_{\mathrm{C} 3} /\left(M_{\mathrm{C} 0}+M_{\mathrm{C} 9}\right)\right)$ obtained for $i_{\mathrm{o}}=8^{\circ}$ and $T_{\mathrm{p}} / T_{\mathrm{b}}=10$. The mass ratio $M_{\mathrm{C} 3} /\left(M_{\mathrm{C} 0}+M_{\mathrm{C} 9}\right)$ is $M_{\mathrm{C} 3} /\left(M_{\mathrm{C} 0}+M_{\mathrm{C} 9}\right)<0.1$, i.e., the mass of the $\mathrm{BH}$ associated with component $\mathrm{C} 3$, is far lower than the mass $M_{\mathrm{C} 0}+M_{\mathrm{C} 9}$.
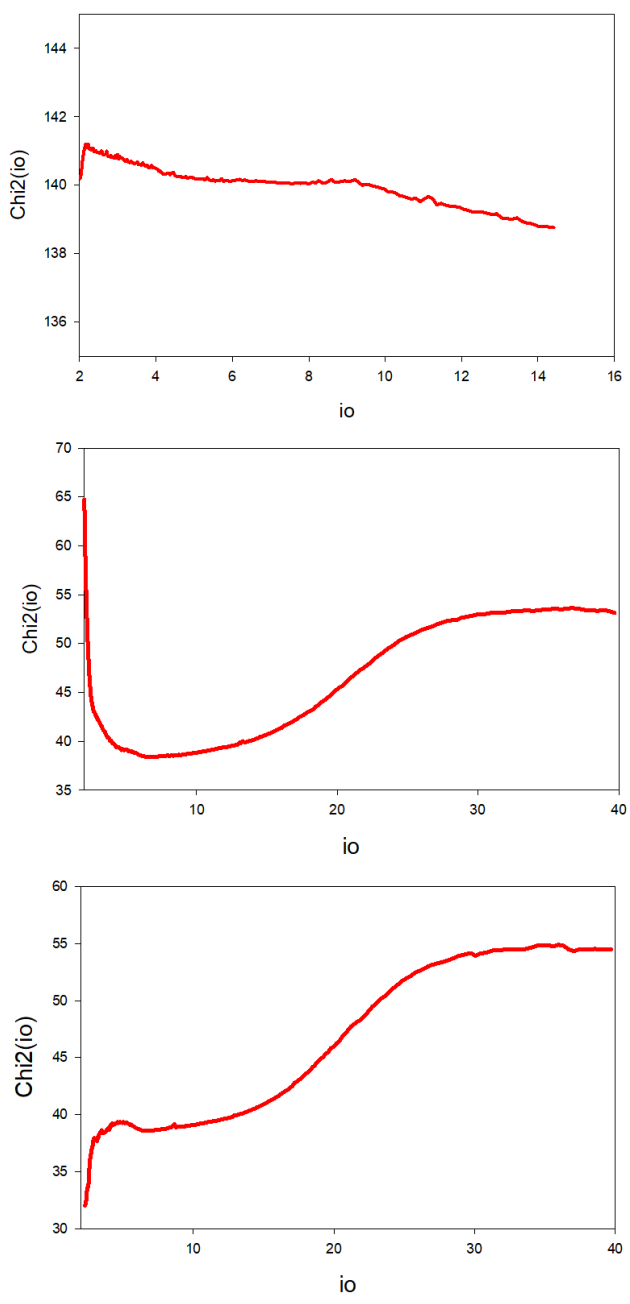

Fig. D.2. Top: precession model. Component $\mathrm{C} 7$ is ejected from the core $\mathrm{C} 0$ : the function $\chi^{2}\left(i_{0}\right)$. The curve $\chi^{2}\left(i_{0}\right)$ is convex and it does not have a minimum, i.e., there is no stable solution. Middle: $\mathrm{BBH}$ model $(\mathrm{C} 0+\mathrm{C} 9)-\mathrm{C} 3$ characterized by $R_{\mathrm{G}-\mathrm{C} 3}=0.37$ mas and the mass ratio $M_{\mathrm{C} 3} /\left(M_{\mathrm{C} 0}+M_{\mathrm{C} 9}\right)=0.01$. Component $\mathrm{C} 7$ is ejected from the component C3: the function $\chi^{2}\left(i_{\mathrm{o}}\right)$. The curve $\chi^{2}\left(i_{\mathrm{o}}\right)$ has a minimum, $\chi_{\min }^{2} \approx 38.4$ at $i_{\mathrm{o}} \approx 6.9^{\circ}$. Bottom: $\mathrm{BBH}$ model $(\mathrm{C} 0+\mathrm{C} 9)-\mathrm{C} 3$ characterized by $R_{\mathrm{G}-\mathrm{C} 3}=$ 0.37 mas and the mass ratio $M_{\mathrm{C} 3} /\left(M_{\mathrm{C} 0}+M_{\mathrm{C} 9}\right)=0.05$. Component $\mathrm{C} 7$ is ejected from the stationary component $\mathrm{C} 3$ : the function $\chi^{2}\left(i_{\mathrm{o}}\right)$. The curve $\chi^{2}\left(i_{0}\right)$ does not have a minimum, i.e., there is no stable solution. 


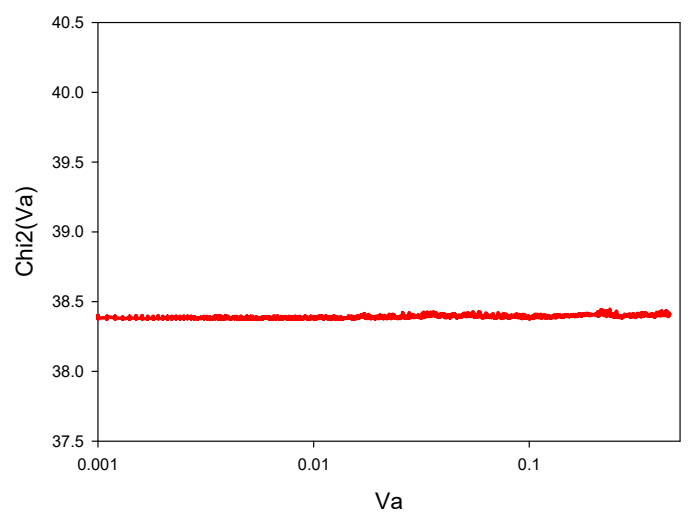

Fig. D.3. $\mathrm{BBH}$ model $(\mathrm{C} 0+\mathrm{C} 9)-\mathrm{C} 3$ characterized by $R_{(\mathrm{C} 0+\mathrm{C} 9)-\mathrm{C} 3}=$ 0.37 mas and the mass ratio $M_{\mathrm{C} 3} /\left(M_{\mathrm{C} 0}+M_{\mathrm{C} 9}\right)=0.01$. Component $\mathrm{C} 7$ is ejected from the stationary component C3: the function $\chi^{2}\left(V_{\mathrm{a}}\right) \cdot \chi^{2}\left(V_{\mathrm{a}}\right)$ remained constant, indicating a degeneracy of the solution.

have a minimum, however, and when $i_{\mathrm{o}}$ becomes smaller than $4.8^{\circ}$, the radius of the accretion disk diverges and becomes larger than the separation of the BBH system (see Fig. D.2).

We adopted the solution with $M_{\mathrm{C} 3} /\left(M_{\mathrm{C} 0}+M_{\mathrm{C} 9}\right)=0.01$ and $R_{\mathrm{G}-\mathrm{C} 3}=0.37$ mas, which corresponds to the best solution. The corresponding curve $\chi^{2}\left(i_{\mathrm{o}}\right)$ is shown in Fig. D.2. The solution is characterized by an inclination angle $i_{\mathrm{o}} \approx 6.9^{\circ}, T_{\mathrm{p}} / T_{\mathrm{b}} \approx 10.7$, and $\Omega \approx 3.8^{\circ}$. The value at the minimum $\chi_{\min }^{2} \approx 38.4$ is lower than the value of the minimum of $\chi^{2}\left(i_{\mathrm{o}}\right)$ when component $\mathrm{C} 7$ is ejected by the $\mathrm{BBH}$ system $\mathrm{C} 0-\mathrm{C} 9$ from the core $\mathrm{C} 0$ (see Fig. D.4).

Using the parameters of the solution, we gradually varied $V_{\mathrm{a}}$ between $0.001 c$ and $0.45 c$. The function $\chi^{2}\left(V_{\mathrm{a}}\right)$ remained constant, indicating a degeneracy of the solution (see Fig. D.3). The corresponding ranges of the parameters of the $\mathrm{BBH}$ system are given in Sect. 3.6.

\section{D.3. $\mathrm{BBH}$ model $\mathrm{CO}-\mathrm{C9}$ : origin $\mathrm{CO}$}

In this section we model the ejection of the VLBI component $\mathrm{C} 7$ assuming that it is ejected by the same $\mathrm{BBH}$ system that has ejected $\mathrm{C6}$, that is, the $\mathrm{BBH}$ system $\mathrm{C} 9-\mathrm{C} 0$, whose separation is

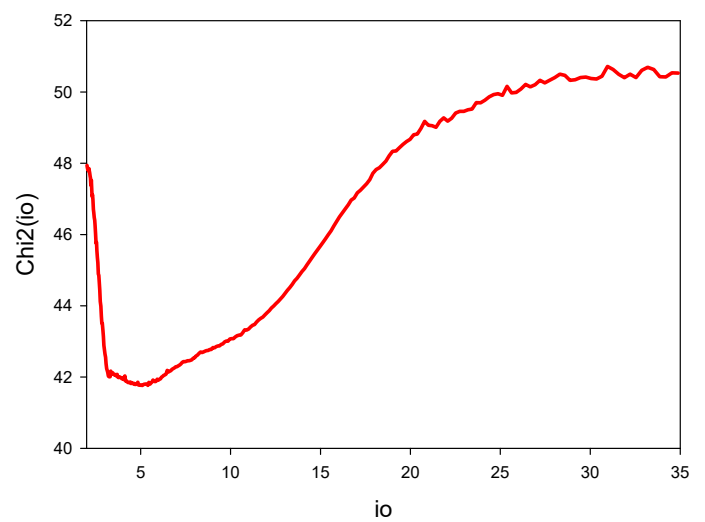

Fig. D.4. $\mathrm{BBH}$ model $\mathrm{C} 0-\mathrm{C} 9$ characterized by $R_{\mathrm{C} 0-\mathrm{C} 9}=0.3$ mas and the mass ratio $M_{\mathrm{C} 0} / M_{\mathrm{C} 9}=0.5$. Component $\mathrm{C} 7$ is ejected from the core $\mathrm{C} 0$ : the function $\chi^{2}\left(i_{\mathrm{o}}\right)$. The curve $\chi^{2}\left(i_{\mathrm{o}}\right)$ has a minimum, $\chi_{\min }^{2} \approx 41.8$ at $i_{\mathrm{o}} \approx 5.0^{\circ}$. The solution is characterized by $T_{\mathrm{p}} / T_{\mathrm{b}} \approx 9.3$ and $\Omega \approx 1.2^{\circ}$ and is rejected because its geometrical parameters are different from those of the BBH system $\mathrm{C} 0-\mathrm{C} 9$ ejecting $\mathrm{C} 6$.

$R_{\mathrm{C} 0-\mathrm{C} 9}=0.3$ mas, and the mass ratio is $M_{\mathrm{C} 0} / M_{\mathrm{C} 9}=0.5$. The VLBI ejection is perturbed by the precession of the accretion disk and the motion of the BHs, and the ejection origin is core $\mathrm{C} 0$. The directions for the precession rotation and the $\mathrm{BBH}$ rotation are chosen to be + and - , respectively.

First, we calculated the function $\chi^{2}\left(M_{\mathrm{C} 0} / M_{\mathrm{C} 9}\right)$ for $i_{\mathrm{o}}=8^{\circ}$ and $T_{\mathrm{p}} / T_{\mathrm{b}}=10$. The solutions corresponding to the ejection of C7 are characterized by $M_{\mathrm{C} 0} / M_{\mathrm{C} 9}<1.0$.

The curve $\chi^{2}\left(i_{\mathrm{o}}\right)$ corresponding to the mass ratio $M_{\mathrm{C} 0} / M_{\mathrm{C} 9}=$ 0.5 is shown in Fig. D.4. The curve $\chi^{2}\left(i_{0}\right)$ has a minimum, $\chi_{\min }^{2} \approx 41.8$ at $i_{\mathrm{o}} \approx 5.0^{\circ}$. The solution is characterized by $T_{\mathrm{p}} / T_{\mathrm{b}} \approx 9.3$ and $\Omega \approx 1.2^{\circ}$. These values should be the same as those found for the solution corresponding to the ejection of C6 by $\mathrm{C} 0$, that is, $\left(T_{\mathrm{p}} / T_{\mathrm{b}}\right)_{\mathrm{C} 6} \approx 5.5$ and $\Omega_{\mathrm{C} 6} \approx 3.1^{\circ}$. When $\mathrm{C} 0$ ejects different components, they are characterized by the same geometrical parameters $T_{\mathrm{p}} / T_{\mathrm{b}}$ and $\Omega$. This solution is therefore not the correct one and is rejected. Its $\chi_{\text {min }}^{2} \approx 41.8$ is larger than the $\chi_{\min }^{2} \approx 38.4$ of the solution obtained for the $\mathrm{BBH}$ system $(\mathrm{C} 0+\mathrm{C} 9)-\mathrm{C} 3$ (see Appendix D.2). 